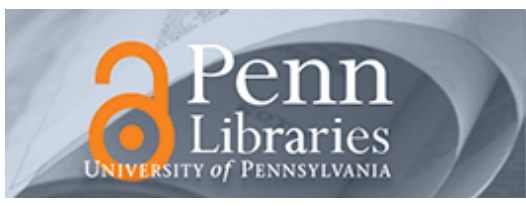

University of Pennsylvania ScholarlyCommons

1983

\title{
Some Major Theoretical Problems Concerning the Concept of Hierarchy in the Analysis of Tonal Music
}

Eugene Narmour

University of Pennsylvania, enarmour@sas.upenn.edu

Follow this and additional works at: http://repository.upenn.edu/music_papers

Part of the Musicology Commons, and the Music Theory Commons

\section{Recommended Citation}

Narmour, E. (1983). Some Major Theoretical Problems Concerning the Concept of Hierarchy in the Analysis of Tonal Music. Music Perception: An Interdisciplinary Journal, 1 (2), 129-199. http://dx.doi.org/10.2307/40285255 


\title{
Some Major Theoretical Problems Concerning the Concept of Hierarchy in the Analysis of Tonal Music
}

\begin{abstract}
Level-analysis in the field of music theory today is rarely hierarchical, at least in the strict sense of the term. Most current musical theories view levels systemically. One problem with this approach is that it usually does not distinguish compositional structures from perceptual structures. Another is its failure to recognize that in an artifactual phenomenon the inherence of idiostructures is as crucial to the identity of an artwork as the inherence of style structures. But can the singularity of an idiostructure be captured in the generality of an analytical symbol? In music analysis, it would seem possible provided closure and nonclosure are admitted as simultaneous properties potentially present at all hierarchical levels. One complication of this assumption, however, is that both network and tree relationships result. Another is that such relationships span in both "horizontal" (temporal) and "vertical" (structural) directions. Still another complication is the emergence of transient levels. In this paper, a tentative solution to these problems is offered by invoking a hypothetical theory that relies on the cognitive concepts of return, reversal, and continuation (i.e., similarity) as regards the parameters of melody, harmony, and duration. Applied to the theme of Mozart's Piano Sonata, K. 331, this analytical theory is contrasted with several systemic analyses of the same theme by the theorists DeVoto, Lester, Schenker, and Meyer. In conclusion, the hierarchical analysis of the Mozart theme gives way to a synthesis as the melody's various levels are rendered into rankings of pitch shown on one level only.
\end{abstract}

\section{Disciplines}

Music | Musicology| Music Theory 


\title{
Some Major Theoretical Problems Concerning the Concept of Hierarchy in the Analysis of Tonal Music
}

\author{
EUGENE NARMOUR \\ University of Pennsylvania
}

\begin{abstract}
Level-analysis in the field of music theory today is rarely hierarchical, at least in the strict sense of the term. Most current musical theories view levels systemically. One problem with this approach is that it usually does not distinguish compositional structures from perceptual structures. Another is its failure to recognize that in an artifactual phenomenon the inherence of idiostructures is as crucial to the identity of an artwork as the inherence of style structures. But can the singularity of an idiostructure be captured in the generality of an analytical symbol? In music analysis, it would seem possible provided closure and nonclosure are admitted as simultaneous properties potentially present at all hierarchical levels. One complication of this assumption, however, is that both network and tree relationships result. Another is that such relationships span in both "horizontal" (temporal) and "vertical" (structural) directions. Still another complication is the emergence of transient levels. In this paper, a tentative solution to these problems is offered by invoking a hypothetical theory that relies on the cognitive concepts of return, reversal, and continuation (i.e., similarity) as regards the parameters of melody, harmony, and duration. Applied to the theme of Mozart's Piano Sonata, K. 331, this analytical theory is contrasted with several systemic analyses of the same theme by the theorists DeVoto, Lester, Schenker, and Meyer. In conclusion, the hierarchical analysis of the Mozart theme gives way to a synthesis as the melody's various levels are rendered into rankings of pitch shown on one level only.
\end{abstract}

$\mathrm{T}$ HE concept of hierarchy reverberates these days throughout the academic world. Neither scholarly nor scientific literature has escaped its influence. Perhaps no concept in the history of ideas has ever cut across quite so many different intellectual disciplines. As a mode of explanation, it has permanently altered the analytical methodology and epistemological outlook of researchers everywhere.

Eugene Narmour is associate professor and chairman of the Department of Music at the University of Pennsylvania. He is the author of Beyond Schenkerism: The need for alternatives in music analysis. Currently he is writing a book on music analysis that explores the implication-realization model.

Requests for reprints may be sent to Eugene Narmour, Department of Music, University of Pennsylvania, 201 S. 34th Street D8, Philadelphia, Pennsylvania 19104. 
A marriage of necessity between rationalism and empiricism gave birth to the idea of hierarchies. Empirical research in this century caused the recognition that natural and artifactual phenomena are more inherently complex than ever before dreamed. As a consequence, hypothetical theories became increasingly rationalistic with the paradoxical result that the number of "givens" necessary for analysis at every operational stage tended to multiply, thereby adding complexity to the observations. In short, rationalism and empiricism eventually found themselves entangled in the same epistemological bed. ${ }^{1}$

The offspring of this entanglement was the idea of hierarchy. For by analyzing phenomena in terms of levels of meaning, the concept of hierarchy seemed to cut through the growing complexity of our theories, promising to restore balance to rationalism and empiricism in the making of analytic theory. Hierarchy did not just emerge as an inevitable cognitive mode for intellectual inquiry, however. Levels also seemed an essential empirical property of both scientific and artifactual objects. Observing adjacent and nonadjacent relationships functioning within levels and between levels appeared to bring into focus what otherwise materialized as a blur.

One reason for the growing complexity of human knowledge was the inescapable empirical conclusion that time is an actively shaping, fundamental variable inherent in all natural and artifactual phenomena-rather than just an idealized, passive medium within which events occur. Nowhere is this more true than in artifactual phenomena where nonrecurrent psychological time must be seen as a virtual property. In a poem, for instance, or a dance or a film or, most tellingly, in a musical composition, the learned and inborn temporal workings of each individual human mind actively participate in the stylistic and idiostructural creation of the artwork.

Thus, by enabling us to deal empirically with musical functions of everincreasing number and complexity, the concept of hierarchy in this connection appeared to offer the only rational way to come to grips with the slippery factor of nonidealized time. But as shall be seen, in the absence of a secure theory of cognition and perception, the concept of hierarchy-because of its inestimable heuristic potential and great analytic utility-forces a number of fundamental questions upon us about the nature of analyzing musical levels.

\section{Boundaries of Levels: Compositional Structures vs. Perceptual Structures}

To begin with, merely identifying the boundaries where level-events are likely to take place-a methodological precondition for theorizing about

1. On the blurring of rationalism and empiricism, see Quine (1963). 
the functional interrelationships of hierarchies-is often a difficult task in analyzing a piece of music. The relative duration of any given note, for example, presumably the very stuff of time, does not necessarily offer a clue as to the parsing of levels. Nor does the highest note in a melodic phrase or the most consonant note in a harmonic progression always indicate where levels begin or end. In short, locating the "natural" boundaries of musical levels turns out to be problematic.

Analysts attempted to deal with this in several ways. One was to identify level-boundaries according to the similarities or differences of form that seemed to appear visually in the notation of the score. Another was to fix level-boundaries according to the combined pitch-collections of melody and harmony that seemed to delineate various key relationships. The former method led to a taxonomy of forms of various temporal "sizes" (motives, phrases, periods, etc.) while the latter brought about the functional analysis of melody and harmony through the chordal successions underlying the defined keys.

Both these programs met with difficulty. The analytical results of one approach often displayed no correlation to the analytical results of the other. Changes in key (presumably marking off the end of one level and the beginning of another) often occurred right in the middle of what otherwise appeared to the eye to be one unbroken form in the score. Also, within each approach there was insufficient agreement among the practitioners as to the formulation of the theoretic-analytic rules-with the usual attendant epistemological lack of consistency, generalizability, parsimony, and so forth.

In the absence of such rules, many different camps utilizing one or some combination of both these programs sprang up, each asserting its own rationalistic theory. What started off basically as two empirical programs thus ended up with different "schools" espousing their own technique of identifying the location of level-boundaries. Among the formalists, for example, there was the wide variety of methodology characterized by the analytical work of such diverse researchers as Reti, Cooke, Cone, Fischer, and others. Among key-functionalists like Riemann, Tovey, Piston, Schenker and his disciples, the same analytic diversity prevailed. ${ }^{2}$ Consequently, the final argument for one school's approach over another ultimately boiled down to the ontological statement, "this is the way people hear it,"-in other words, a rationalistic fallback to the predefined ideal of a "competent listener."

But those discontent with this rationalistic retreat-chiefly the historical musicologists and the historians of music theory-had another approach in

2. More than the others, the Schenkerians, of course, see differentiating key relations as underscoring a unified tonal scheme. 
mind. They believed that level-boundaries could be deduced from discovering compositional structures. Indeed, many recent theoretical approaches to hierarchical analysis in tonal music depend directly or indirectly on a knowledge of compositional schemata as their analytical point of departure. For example, scholars and theorists look to the compositional manuals and instruction books of a given period as a way of trying to learn what a composer might rely on in terms of compositional plan since presumably every composer "reflects" his environment and is thus to some extent a product of his educational background. To take another example, one surmises that the rapidly expanding and popular field of deciphering compositional sketches came about partly because scholars felt this activity seemed to hold great hope for discovering compositional schemata with all that apparently promised for identifying both the low- and high-level patterns of a given stylistic context. ${ }^{3}$ The notion of compositional structures, of course, permeates the analytical field of atonal music theory (twelve tone, serial, set theoretic, etc.) because the composers originating the style (Schoenberg, Berg, and Webern) preoccupied themselves with precompositional, protostructural determinations.

Whether gleaned from sketches or instruction books, compositional strategies are, of course, obviously interesting in and of themselves and unquestionably possess great utilitarian value for musicological and theoretical study. However, it gradually became clear that all these attempts to solve the problem of locating level-boundaries raised a fundamental question: in any explanation of how a given piece of music is comprehended, is one justified in believing that compositional structures indicate the boundary locations of perceptual hierarchical levels? Analytically, can we rely on the assumption that overt compositional plans translate directly into perceptual-cognitive structures?

It is, of course, one of our fondest aesthetic-cultural beliefs-traceable probably to the Romantic view of the artist-as-priest-that a one-to-one relationship exists between what a composer intends and what a listener hears. But there is no a priori reason whatever to believe that the correspondence between compositional structures and perceptual structures is a constant one. Indeed, the perceptual "interface" between a given composer's music and the listener is one of the most transitory and fluctuating relationships in the whole domain of human communication. Because a composer carefully plans out a piece in terms of what he thinks are structural relationships by no means ensures that these relationships will be perceived as such.

3. For discussion of the logical problems involved in the "contextual" approach, see Ellis (1974), particularly chapter 5 . 
A composer may lay out in advance the key scheme of a development section of, say, some 200 measures he plans to compose. But once composed, the key scheme spanning such a period of time may possess no structural significance whatever from the cognitive-perceptual vantage point.

Moreover, though it may be analytically useful to parse a piece into twenty or thirty hierarchical levels, a listener may only keep track of three or four of those levels at any one time-and not necessarily the same three or four levels throughout the piece. The levels a listener attends to simply may not coincide with the continuous, consecutive order of levels in our analysis. As empirical studies in cognitive psychology of the last fifty years conclusively demonstrate, the human cognitive apparatus has its own peculiar way of structuring and comprehending the world which may bear little or no resemblance to rationalistic-hypothetical plans of either composition or analysis.

As analysts, furthermore, in moving from lowest level to highest level in a large, many-leveled hierarchy, it is hard not to believe in the face of our analytical experience that a crossover region exists where we find ourselves gradually shifting interest from what appear to be perceptual structures to what are manifestly rationalistic compositional structures. This is why the operation of recursion with respect to the analytical rules of a given hierarchy theory is so problematic; even if consistent generative results could be discovered, we would have to be suspicious of them in view of the compositional-perceptual dichotomy.

How to define this threshold in any given piece and thus prevent confounding the discontinuous aspects of composition and perception in the identification of level-boundaries is therefore a major problem for both music theory and cognitive psychology. ${ }^{4}$ For whatever the long-term value of the rationalistic theories which depend on compositional structures and which dominate the field of music analysis today, hierarchical relationships also exist empirically as psychological facts of cognition and perception. ${ }^{5}$ Furthermore, these latter relationships often appear nonveridical and incommensurate with the level-boundaries derived from compositional structures. Separating rationalistic compositional-structural hierarchies from

4. Since learning is irreversible, analysis by itself is little help in solving this problem: on discovering a compositional structure, a music theorist easily comes to hear it-and thus believes it to form an intrinsic part of the original experience. What starts out as an "inaudible" (compositional) form very quickly takes on the reality of a perceptual one.

5. I am thinking here of the experiments in memory encoding, memory retrieval, list learning, story reconstruction, problem solving, and so forth, not to mention computer simulations and theories of instruction that demonstrate the hierarchical workings of the human brain. 
empirically verified perceptual ones thus presents a major difficulty neither music theory nor cognitive psychology can ignore since it is the interrelationship between these two types of hierarchical generations that ultimately determines the aesthetic evaluation of the musical artwork.

\section{Idiostructures vs. Style Structures}

Still another major difficulty with the analysis of musical hierarchies is that of distinguishing idiostructures from style structures. ${ }^{6}$ Because we lack a general agreement as to what the definition of an idiostructure is, this problem is even less well-understood than the relatively difficult one of separating compositional structures from perceptual structures. But it is a crucial issue, for it takes us to the heart of the matter of what the concept of hierarchy means with respect to analyzing and explaining artifactual phenomena.

It is widely thought, for instance, that the idiosyncratic elements of a musical artwork take place primarily on lower levels. ${ }^{7}$ Thus, a simplistic analytical "flowchart" of how such an approach would deal with the style structure and idiostructure of a piece of music would look something like this:

(1)

low level
(surface; foreground)
middle level
(middle ground)
high level
(background)

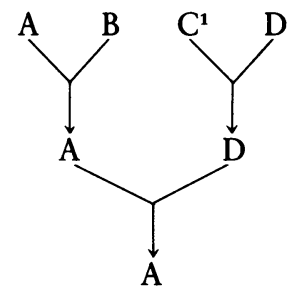

-where the abstractions A, B, C, and D originating from a pool of known style structures would generate the level-transformations while uniqueness (occurring, say, at C and symbolized by the superscript " 1 ") would function as an idiosyncratic event. Thus, the closer the analytical levels are to the high-level background, the more stylistically alike all pieces will appear to be. Of course, this type of level-generation, which systematically filters out uniqueness as the analysis progresses from lower levels to higher levels,

6. I have discussed this in some detail in chapter 11 of my book (Narmour, 1977). By "style structure" I essentially refer to the relationships of closed parametric complexes that are replicated within and between pieces. By "idiostructure" I refer to those relationships of closed parametric complexes that are not replicated.

7. Schenkerian theory, for example, espouses this view-implicitly if not explicitly. 
could hardly view idiostructure as residing anywhere else but close to the surface foreground since the analytical reductions are generated in the first place from a preassembled, a priori collection of presumably known transforms - that is, from the numerous structures hypothesized to make up the style.

Now in theories exemplifying such transformational reductionism, the idiostructural function of any given pitch is explained by invoking all of its level-connections simultaneously, from the lowest to the highest, since such concrete differentiation will minimize repetition and recurrence and thus stylistic sameness or similarity from piece to piece. That is, in the example just given, the unique event, "superscript 1 ," would be explained in terms of the total context of: the contiguous events of $\mathrm{B}, \mathrm{C}$, and D on the surface level, the events of $A$ and $D$ on the middle level, and finally the event $A$ on the highest level.

To be sure, few scientists would quibble with this approach since generalizations (read style) are known to illuminate the meaning of uniqueness when universality of explanation is the epistemological goal. However, the philosophical difficulty with this explanation of uniqueness for the humanist-and the classical objection toward having faith in such "contexting"is that it reduces an idiostructural event to a default case of the style. And this runs completely counter to our aesthetic experience since those things that essentially characterize artworks and that collectively create their highly idiosyncratic nature can hardly be rationally thought of as surface anomalies or ornamental aberrations. Whatever is essentially unique in an artwork does not present itself to us as a negative occurrence dependent on prior generalization for meaning. Rather, idiosyncracy comes to us as an independent and positively structured event.

There is, however, an even more serious operational objection to such contexting explanations of idiostructures-one which hierarchy as a concept of partially decomposed levels forces us to deal with. ${ }^{8}$ And that is this: in a true hierarchical concept of levels, as opposed to a systems view of levels, whatever happens on the lowest level becomes permanently embodied in the next level and thus continues to influence that level regardless of how generalizable the new level appears to be. This means in our hypothetical diagram, for example, that uniqueness on the low level must affect transformation on the next level. That is, the uniqueness of $\mathrm{C}^{1}$ will "infect" its contiguous surroundings $\mathrm{B}$ and $\mathrm{D}$ such that it inheres in the neighboring events and thus is carried to all of the next levels. For in a true hierarchy, the effects of events from one level to the next are not screened out but rather

8. On the "partial decomposability" of hierarchies, see Simon (1969), chapter 4 especially. 
remain an inherently active part of the transform. If this were not so, we would not speak of hierarchical levels as being partly decomposable. A revised hierarchical diagram of our earlier example would thus look something like this (where the uniqueness of $\mathrm{C}$ must be conceived as ultimately affecting every part, as symbolized by the broken lines):

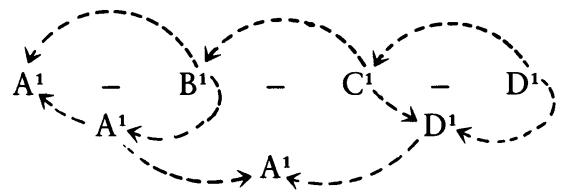

This approach, however, which admits idiostructure as a phenomenon independent of style and which asserts hierarchical levels as partly decomposable units, is as unsatisfying as our earlier systemic example where uniqueness functioned dependently in the service of style since here every event appears more or less unique. In other words, in this hierarchical approach, we would be viewing style as a default case of idiosyncrasy. Every single event and combination of events would be absolutely unique; and similarity between pieces would have to be conceived as anomalous. Variation as an independent empirical observation of repetition would be rendered irrelevant.

It is peculiar-particularly in the humanities with its emphasis on single artworks-that the view of idiostructure as a default case of style goes so unchallenged whereas in contrast the view of style as an aberration of uniqueness is quickly derided. But the latter is no more or less absurd than the former vis-à-vis our experience of musical artworks-indeed, both exemplify what might be called the "fallacy of reduction."

Stylistic sameness or similarity; idiosyncratic difference or singularityboth are perceptual facts of life. We emphasize one or the other, depending on whether we are adopting a "scientific" or a "critical" approach in analyzing a musical phenomenon. But both always operate cognitively in parallel, independent and separate, yet always correlated to a greater or lesser degree. Because this is so, the matter of hierarchical analysis in music becomes much

9. An analytical reduction can never completely represent a given level. Assuming that criticism is a major goal of music theory and thus that uniqueness must be explained, even an elaborate tranformational reduction will let crucial structural information slip through the analytic-explanatory net. As sophisticated as Schenkerian theory seems to be, for instance, its analytical mesh is still much too coarse to catch the critical idiostructures of melody, rhythm, form, etc., because its symbology is too grossly reductive to allow an interpretation of the anlysis to be rendered into a consistent theory of criticism in natural language. One problem obviously feeds back into the other: a deficient analytical symbology leads to erroneous conclusions about hierarchical levels, while an epistemological misconception about hierarchical levels, as they pertain to the idio- and style-structural aspects of musical artworks, prevents us from formulating better theories of music. 
more complicated. For it means we must develop theories that admit style and idiosyncrasy as being simultaneously present on every given level and at any given moment in musical time.

The problem of not coming to grips with the matter of idiostructure in the analysis of musical artworks is partly the result of a general misconception about how levels function in a true hierarchy.$^{10}$ For, as we shall now discuss further, a fundamental tenet of hierarchical theory, as opposed to systems theory, is that hierarchical levels can only be partly disassembled. This means that a given level must be explained both in terms of itself and the other levels it gives rise to. It also means that the properties of any given level perforce substantively affect the elements of the other contiguous and discontiguous levels it connects with.

\section{"True" Hierarchical Levels vs. Systemic Ones}

It is unfortunate that the word hierarchy has come to refer willy-nilly to all manner of level-analysis. What we need is a specialized conceptual terminology that differentiates true hierarchical levels from systemic ones. For in a real hierarchy, as I have emphasized, a given level is always and only partially decomposable - that is to say, it is in principle unreducible in significant respects to higher levels because the higher-level transformation is composed in part of nonassimilable properties that originate only and uniquely from the lower level. Were this not so-were uniqueness filtered out and left on lower levels as a "residue" as a tone moved to the next given level - then levels in music would be much more amenable to complete decomposition, and traditional reductionism would be the means by which level-interactions would be explained. But if it is true in hierarchies that levels are partially decomposable, then it follows that when uniqueness is a crucial property of a given pitch on a lower level that is transformed to a higher level, then that uniqueness is embodied in that tone and stays with it as it moves to the higher level. This has very important ramifications for the analysis of musical artworks which an example will make clearer.

Suppose, for instance, we had two "melodies" with analyses something like Figure $1 \mathrm{a}$ and $1 \mathrm{~b}$. A typical theoretical observation would draw attention at the second level to the sameness of structure in both melodies

10. In a general sense, both the misconception of hierarchy and the lack of sufficiently refined analytical theories are why musicology, despite more than a century of existence, has yet to yield a significant body of enduring critical studies. Nor will it do so until analytical symbologies are capable to some extent of capturing idiostructures. Until then, critical studies of musical artworks will remain works of "fiction," more to be admired for their own singular literary form than for contributing any lasting arguments about the uniqueness of the musical artworks they purport to deal with. 


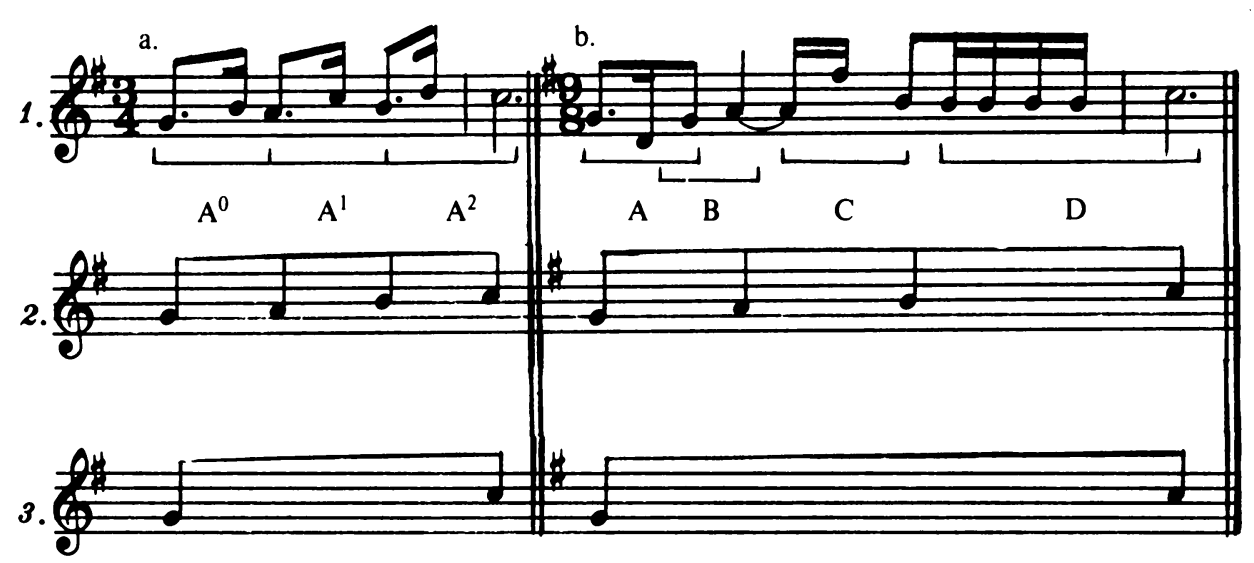

Figure 1.

(namely, the ascending G-A-B-C line), while asserting differentiation between them on the lowest "surface" level, the level of the printed music. And a statement like "both melodies 'compose out' or 'prolong' the same ascending line" would typify the kind of interpretation attached to such an analysis, following quite naturally from the transformationalist belief that music makes its temporal way by essentially combining and recombining, but nevertheless always exemplifying, a preexistent set of transforms, in this case, the ascending line.

Now analytical explanations tied to such an approach are heavily reductionistic. Melodic novelty would be seen in terms of a comparison as to how the low-level sequences of pitch and duration are specifically attached to and differentially deployed in relation to the high-level linear similarity. In other words, the higher-level linear structure-in actuality a style transform-would be brought to bear on the surface of the melodies as a kind of decompositional searchlight, systematically parsing relationships between the pitches so as to illuminate their level-functions. Explanations based on this type of analytical approach are, of course, legion; and they can be found, implicitly or explicitly, on practically every page of analysis published in the last fifty years.

But strictly speaking, analyses like those of Figure $1 \mathrm{a}$ and $1 \mathrm{~b}$ are not hierarchical ones, despite their appeal to, and display of, levels of meaning. Such analyses in fact reflect the epistemological concept of "organized system" or "systemic reductionism," not hierarchy. ${ }^{11}$ For in a true hierarchi-

11. By "systemic" throughout this paper, I mean to refer to musical relationships which are conceived in Gestaltist fashion as parts of a completely integrated whole. 
cal analysis of melodies like Figure $1 \mathrm{a}$ and $1 \mathrm{~b}$, the higher-level similarity of G-A-B-C would have to reflect analytically the precise way in which lowerlevel melodic and rhythmic functions contribute to higher-level differentiation. That is, though in a systemic reductive analysis the levels of Figure 1a and $1 \mathrm{~b}$ look to be the same in view of each generating the same ascending linear pattern, in a hierarchical sense the linear patterns are not the same because they are differentially affected by the individualizing surface features that cause (in part) the high-level transformations in the first place. ${ }^{12}$

In a true hierarchical analysis, the difference, for instance, between the $B$ on the third beat in Figure 1a (brought about, in part, rhythmically by the sixteenth-note $\mathrm{C}$ reversing direction) and the analogous $\mathrm{B}$ in Figure $1 \mathrm{~b}$ (brought about, in part, rhythmically by the sixteenth-note $F$ leaping downward) would have to be accounted for symbologically so that while the transformed linear pitch sequence on the higher level would not be altered, the functional meaning of each B on level 2, not to mention the other pitches as well, would be measurably different. Stated in formal terms, the inherent differences in conformance between Figure 1a, which motivically is a thrice-repetitive $A^{0}+A^{1}+A^{2}$ (see the analysis), and Figure $1 b$, which motivically is a four-part $\mathrm{A}+\mathrm{B}+\mathrm{C}+\mathrm{D}$, must be incorporated into the symbological meaning of the linear structures on the next level. A systemic reductive explanation, no matter how fully dressed in the interpretive clothing of a natural-language explanation, would fail to cover the hierarchical meaning of the examples so long as the two analyses on level 2 appeared symbologically exactly the same. ${ }^{13}$

\section{Artifactual Hierarchies and the Theoretical Possibility of an Idiostructural Symbology}

I stress "symbologically" because theories are revealed most fundamentally through their analytical symbols rather than just through the often quite irrational natural-language interpretations, intuitions, and beliefs that accrete to them over their life. In analyzing the melodies above on level 3, for example, the same observations made earlier would apply: a systemic analysis would symbolize both examples as the same $\mathrm{G}-\mathrm{C}$ whereas in a true hierarchical analysis the low-level difference between the two examples

12. I say "in part" because the sources of transformation in a field like music are both external and internal with respect to style, and they are perceptually dependent on both learning and inborn cognitive constants.

13. I would go further and say that any theory lacking an expressed or implied analytical symbology is no theory at all-which is why much that goes on in humanistic fields is of limited value since arguments and hypotheses often never reach any concrete logical formulation. 
would be incorporated in the G-A-B-C lines such that on the third level the G-C pattern would also be differentiated symbologically. Such differentiation is necessary, it should be emphasized, not only to understand the idiostructural separation between the phrases but also to comprehend the style-structural differentiation. Just how both kinds of relationships between the two examples could be theoretically, analytically, and symbologically accounted for is, of course, beyond the scope of the present paper. The fact is, we currently have no analytical music theory capable of dealing symbologically with true hierarchical interactions. ${ }^{14}$

It is worth discussing why this is so, why music theorists-indeed all those professionally concerned with the humanities whose artifactual subject matter is inherently and irreducibly idiostructural-remain so reluctant to embrace the concept of a true hierarchy, one where all the low-level functions bringing about the next-higher level not only contribute to but inhere in the transformation they create. ${ }^{15}$ One probable reason is that in the face of the thousands of unique artworks that beg for explanation, specifying hierarchical individuation seems completely adverse to the idea of generality inherent in the idea of analytical symbol. In invoking a true hierarchical concept in a humanistic field like music, where differentiation is the norm both in terms of style and idiostructure, do we not, for instance, run the risk of fatally obscuring, perhaps permanently losing, the powerful generality of analytical symbols, which is the analytic raison d'être for them in the first place? Is not symbological generality incompatible with the hierarchical demand that the analytical levels explicating artifactual subject matter be idiostructural? Must not criticism and idiostructure always remain within the more congenial explanatory realm of natural language with all its rich interpretive resources of multiplicity of meaning?

The answer to all these questions is: not necessarily. Were true hierarchical levels not a perceptual and phenomenological fact of reality, we could scarcely apprehend or understand the world of idiosyncrasy at all-musical or otherwise. And from what we know about the economy of nature, we have strong reason to believe that the perception and comprehension of uniqueness probably depend on a few general cognitive mechanisms (including ones capable of generating all manner of individuated learning). It follows that those psychological mechanisms must reflect universal laws of some sort. Radical though it may seem, it follows further that these general laws must imply the epistemological possibility of an analytical symbology

14. I have suggested a tentative solution to this problem in my article (Narmour, in press).

15. To reduce these effects by filtering them out of the transformation or to explain them only in terms of a totality of level-connections is insufficient. For more discussion on this point see Pattee (1973, pp. 148-150). 
that will yield unique representations each time the symbology is applied to the analysis of an artwork.

For despite the symbological complexity that would obviously obtain in a truly hierarchical analytical theory applied to inherently idiostructural phenomena, the analytical alternative of generating levels through a theory of systemic reductionism carries a greater risk: that of producing naive, simplistic, fanciful, misleading, or highly distorted analyses and explanations. Even the aspect of style is always differentiated on some level; repetition invariably has the simultaneous property of sameness and similarity; when it occurs, style emerges both as an exact formal replication and an individuated style-structural variation. Thus, a cardinal advantage of creating a real hierarchical theory of music-one where the relevant properties of low-level events inhere virtually in the events of the next-level transforms-is that it would allow us for the first time to deal with style-structural and idiostructural complexities on their own terms, to create a criticism based on logical argument rather than on mere opinion. Of course, to analyze such complexity in the absence of a true hierarchical theory is unthinkable. But more importantly, it is only with the advent of the concept of true hierarchy that we can begin to see how a hierarchical analysis can simplify extreme complexity without distorting it and that the concept of an analytical symbology treating idiostructural uniqueness is not necessarily an epistemological contradiction. ${ }^{16}$

At this point an example is called for. If we had such a hierarchical theory-one where the low-level closural effects generating the next-higher level inhered deterministically in the elements tranformed onto the next level-then the smallest change imaginable between highly similar pieces would have to be dealt with theoretically and symbologically. In melodies like Figure $2 a$ and $2 b$, for instance, the analytical symbology would have to differentiate the high-level ascending linear patterns on the next level even though the patterns of both examples display the form A+A + A and even though both display exactly the "same" systemic level-relation of the G-A$B-C$ pitch sequence on the next level. In other words, the rhythmic difference between the sixteenth-note anacruses in Figure $2 a$ and the thirtysecond-note anacruses in Figure $2 \mathrm{~b}$ would have to be incorporated analytically into the transformed notes on the next level, symbolizing that the notes $\mathrm{A}-\mathrm{B}-\mathrm{C}$ in Figure $2 \mathrm{~b}$ are more closed on the level of their occurrence than the analogous notes in Figure 2a. ${ }^{17}$ Furthermore, such an analytical theory

16. One of the great disciplinary goals of the humanities, in my view, should be to develop just such analytical symbologies.

17. All other things being equal, the more cumulative a pitch is in duration, the more closed it is. 


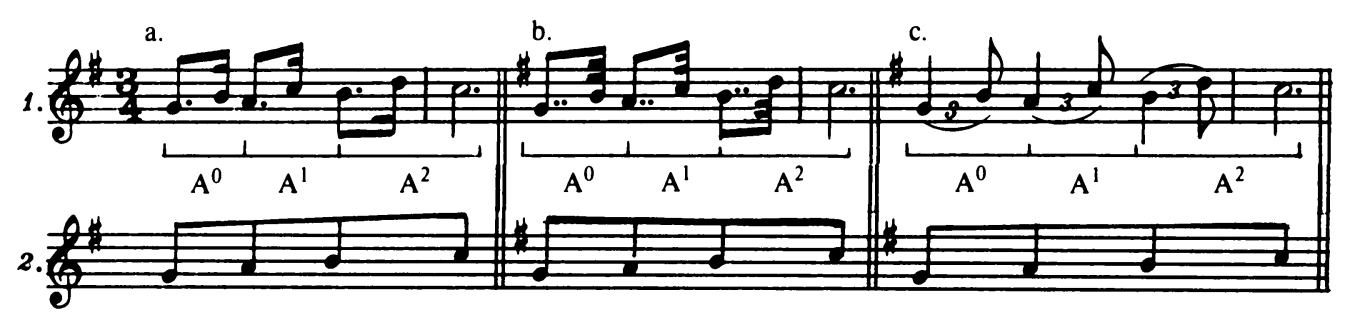

Figure 2.

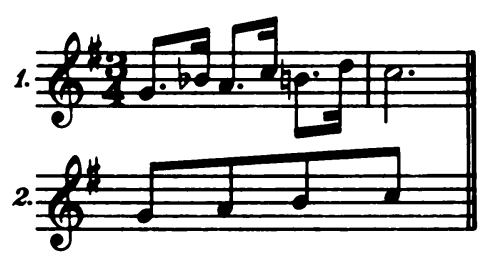

Figure 3.

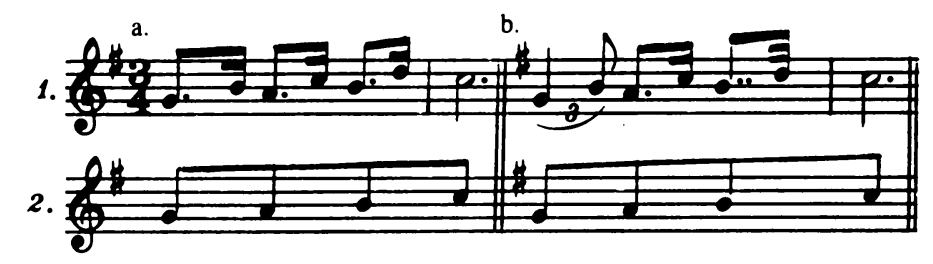

Figure 4.

would have to differentiate symbologically the transformed pitches of a melody like Figure $2 c$ (where rhythmic closure on the $A-B-C$ is weaker) from those of Figure $2 a$ and $2 b$.

Likewise, in a true hierarchy theory the transformed pitches of a melody like Figure 3 would have to symbolize the precise way the $B b$ differentiates the next-level A (beat 2) from the A in melodies like Figure $2 a, 2 b$, and $2 c$. Similarly, were a melody like Figure 4a performed as notated in Figure 4b, with a triplet anacrusis preceding the $\mathrm{A}$ on the second beat (instead of a sixteenth note) and a thirty-second note preceding the terminal C (instead 
of a sixteenth note), the high-level line would have to reflect this differentiation symbologically. ${ }^{18}$

All of this is not to say, of course, that on level 2 of Figures $1 \mathrm{a}, 1 \mathrm{~b}, 2 \mathrm{a}, 2 \mathrm{~b}$, $2 c, 3,4 a$, and $4 b$ the "same" G-A-B-C line does not exist. It is to say, rather, that the higher-level pattern on level 2 among the examples cited is a systemic relation: sameness can be said to pertain only if one views the G$\mathrm{A}-\mathrm{B}-\mathrm{C}$ among the examples as a style form, that is, as one completely decomposable system of sameness, rather than as a style structure, a hierarchically differentiating relation of similarity.

The point is that in a true hierarchical analysis every level always possesses idiostructure (nonrecurrent difference, uniqueness, singularity), style structure (differentiating similarity, i.e., recurrent difference) and style form (exact replication). ${ }^{19}$ Hence, a major problem for music theory in the face of developing a hierarchical theory is to create an analytical symbology that will generate all three relationships: idiostructures, style structures, and style forms. ${ }^{20}$

Let us consider another example. We see that in order for an analysis to qualify as a hierarchical one, the asserted levels must be only partially decomposable, and partial decomposability by definition must refer to level-dependence and level-independence established internally rather than determined only in light of some external, higher-level functional relationship.

For example, the analysis of chord, key, and tonality in a piece via Roman numeral symbology - still the preferred analytical mode in traditional historical musicology despite at least a generation of sophisticated advances in analytic theory-does not yield hierarchical levels. Suppose we were dealing with a piece with a harmonic sequence like Figure 5. As the analysis shows, the different lower-level chord relations that lead to a prolonging of the I and $\mathrm{V}$ chords on level 2 do not translate into any higher-level analytic-

18. In a hierarchical sense, musicologists are thus quite right to worry about the transcription and printed accuracy of the tiniest things in editions of music. And it goes without saying that conductors and performers should also have a genuine hierarchical concern to interpret music as closely as possible to what the composer seems to have intended in the actual notation.

19. That style possesses both replicating and differentiating properties is exemplified in our dualistic use of the term. Sometimes, for instance, we use the word style to refer to the uniqueness of a recurrence. Other times, we employ the term to describe commonality among recurrences. Incidentally, it should be clear once again in light of this discussion that the theoretical concept of idiosyncrasy occurring only on lower levels and style only on high levels-or of perceptual structures occurring only on low levels and compositional structures only on high levels - is not tenable in a true hierarchical theory.

20. I have attempted to explore such a possibility in my paper (Narmour, in press). 

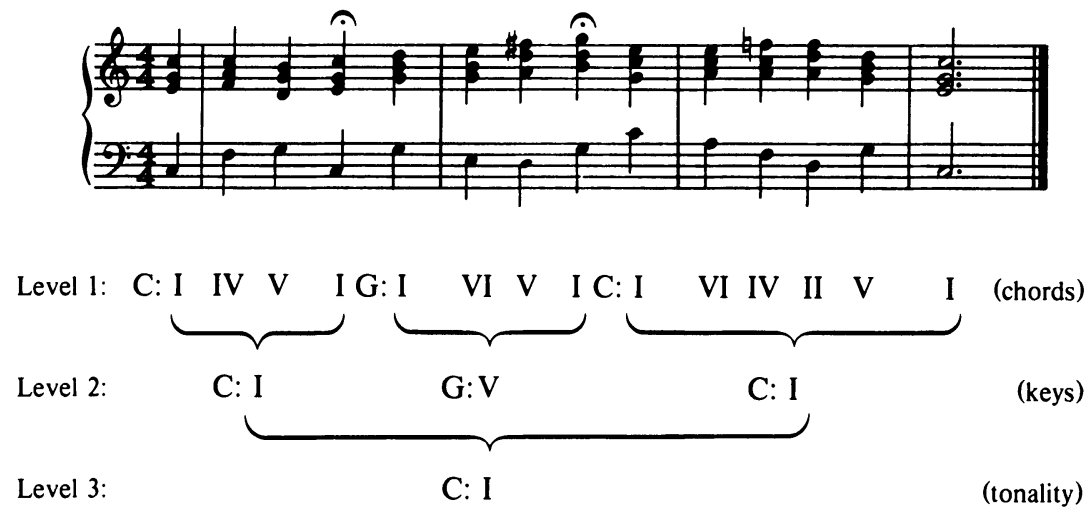

Figure 5.

symbolic differentiation. Typically, nothing that happens internally within the differentiated low-level progressions appears in the analytical symbols to have an effect on the key-structure of level 2 or the tonal structure of level 3. On level 1, the chord differentiation within each bracketed progression (IV-V vs. VI-V vs. VI-IV-II-V), as harmonic motions of seconds, thirds, fifths, or all three, are apparently significant enough to warrant symbological particularization on the low level, but this differentiation disappears completely in the reductive coding on levels 2 and 3.

Explanatorily, we are at the mercy of a loose, nonsymbologically specified interpretation. On level 2, we can understand, for example, the difference between the initial key area of $C$ and the terminal key area of $C$ only by checking back through the system to the lowest level to see how the higher levels are connected. Put another way, because of the nonhierarchical nature of Roman numerals, we can say nothing from the symbols themselves about the internal quality of either of the tonics or the dominant on level 2; nor can we say anything general about the contiguous relations obtaining between the I and the $\mathrm{V}$, and the $\mathrm{V}$ and the I on level 2. Although a systemic level-reduction like this one (with a beginning, a middle, and an end) hardly seems problematic, it does not take much imagination to see that such levelreductions lead quickly to analytical and interpretive difficulties when comparing and contrasting progressions.

Suppose, for instance, the relations of chord and key were exactly as before except this time the phrase ended "openly" on a progression prolonging $G$, the dominant (see Figure 6). Now the parallelism at the second level $(\mathrm{I}-\mathrm{V} / \mathrm{I}-\mathrm{V})$ raises questions both about the quality of the tonic keys on level $2(\mathrm{C}, \mathrm{I})$ and the dominant keys there as well $(\mathrm{G}, \mathrm{V})$. For now we must ask not 

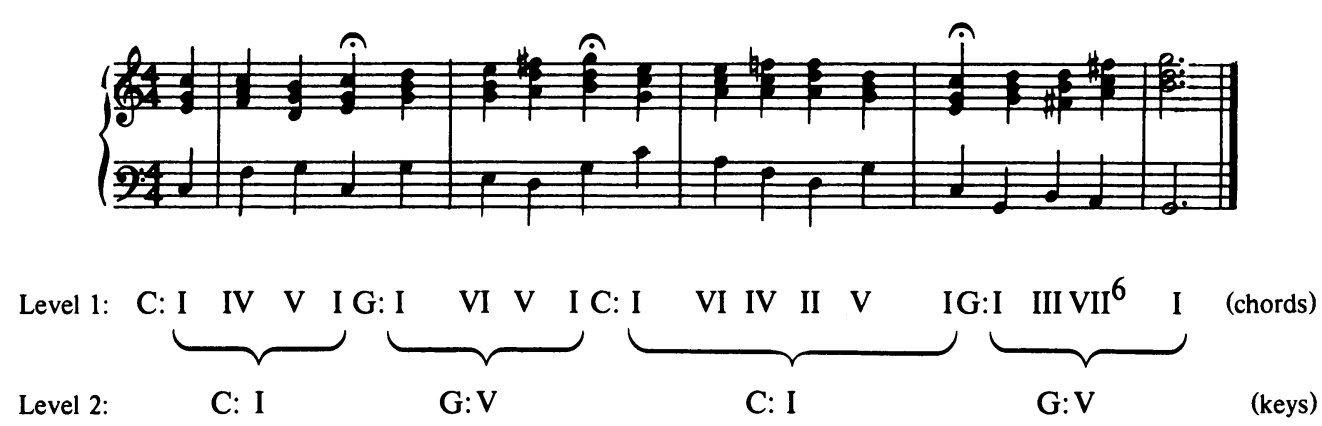

Figure 6.

only how the low-level differentiating progressions contribute internally to each tonic and dominant prolongation on the next level but also how the low-level differentiating progressions contribute to the I-V/I-V key scheme as a whole. For example, is the first dominant on level 2 more "open" than the second by virtue of the tonic system preceding it? That is, though the key scheme I-V/I-V on level 2 looks symbologically analogous, the I-V/I-V keys are by no means parallel hierarchically because of what lies underneath them.${ }^{21}$ And though we might once again attempt to differentiate internally the two V's (or the two I's) on level 2 by verbally interpreting the progressions that contribute to their emergence, such a systemic-reductionist appeal is of little help in determining how the key scheme of $\mathrm{I}-\mathrm{V} / \mathrm{I}-\mathrm{V}$ on level 2 functions in and of itself.

Indeed, in reflecting on the levels hierarchically in this example, as opposed to systemically, it is clear not only that Roman-numeral analytic symbols are deficient in their capability of carrying low-level meanings with them to high levels but that they also are very misleading since any given symbol like "I" on a lower level may have a very different meaning on the next level and even a third meaning on a higher level, owing to the increasing statistical likelihood of prior substratem differentiation.

In a field like music theory whose artifactual subject matter is avowedly idiostructural, a hierarchical theory based on partially decomposable levels would make a severe demand on its analytical symbol-system. But, as we have seen, there are good arguments why an analytical symbology for idiostructures is both necessary and practicable.

For it is the individual composition in all its idiosyncrasy that manifests

21. As I remarked earlier, analysts constantly write systemically about such and such being the same on such and such a level even when it is plain hierarchically that the lowerlevel events underlying such comparisons are manifestly different. 
phenomenological reality. In the world of artworks, uniqueness claims existential priority over all other general properties. It should not be forgotten that the common currencies of a musical artwork-those phenomena we call style forms-are empirical abstractions. Their reality is literally dependent on, and originates from, the existence of individual pieces, and their factuality is always ultimately a matter of theoretical generation.

Thus, any analytical symbology based solely on replicated style forms, which is what all current analytical theories consist of, is epistemologically an abstraction of an abstraction, a generalization of a generalization. This is why present-day theories seem so divorced from musical reality, why present methodologies often seem so devoid of critical illumination, and why current analytical symbologies appear so empty of aesthetic meaning. For the decision to construct music theory from abstract symbological generalizations already based on abstract stylistic generalizations cannot but regress to vacuous rationalism.

Since, as I mentioned earlier, nature seems everywhere to be economical in its fundamental designs, we have good reason to believe that our mechanisms of cognition, perception, and memory, in all their power, probably operate on both idiosyncrasy and commonality according to one parsimonious set of general principles. We recognize the "onceness" of any event in and of itself for what it is at the time in addition to recognizing it through matching and prior experience for what it is and is not. But whatever the psychological mechanisms are, they do not, to repeat, seem to deal with idiosyncratic differentiation as "default cases" of sameness, just as they do not seem to code recurrence as a default case of uniqueness. And if this is true, then there is good reason to think that we could construct an analytical theory grounded on a few general, empirical-presumably psychological or psychophysical-principles to generate idiostructures and differentiating style structures via some idiostructural symbology.

An analogy at this point may be helpful. For the mathematical concept of infinity parallels the humanistic concept of idiostructure. Consider, for example, the symbol $p i(\pi)$, which specifies the ratio of circumference to diameter. Depending on the demands of a given mathematical application, this symbol is rich enough-at least in theory-to yield unique measurement to each and every differentiating case to which it is applied since what lies behind it (and other irrational numbers) is an infinite series of differentiating symbols (in $p i$ 's case, the integer 3 followed by a decimal sequence of nonrecurring digits-3.141592 . . , etc.).

Now suppose, for instance, in the musical analysis of style we hypothesize a class of implicative relationships and use the single symbol " $\longrightarrow$ " to express them. Obviously, such an abstract sign represents class identity (and relational equivalence), but, just as in the decimal representation of $p i$, the 
symbol may also embody degrees of implication. For example, lying behind the general class of implication, an infinite series of degrees of implication could be symbolized from weak to strong as

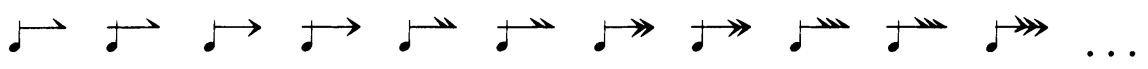

(not necessarily on a linear scale).

In style analysis we might employ only the single arrow symbol to represent the general class of implication-in the same way $\pi$ by itself is useful in analyzing certain kinds of class relationships in mathematics. And in critical analysis we might employ numerous gradated arrow symbols to show varying degrees of implication -in the same way decimal approximations like 3.14 extended to $3.1415,3.14159$ or 3.141592 (and so forth) may be necessary to satisfy the unique demands of certain mathematical applications. (Of course, in a hierarchical, musical analysis the actual number of arrow symbols needed to characterize an idiostructure would be smaller than one might ordinarily think since levels themselves are differentiating.)

Thus, just as there exists one mathematical theory governing both $\pi$ and the infinite decimal system lying behind it, so we may posit the existence of one music theory governing both the class of implication necessary for the analysis of style and the infinite gradations of implication necessary for a fruitful, yet manageable, study of idiostructures.

The radical suggestion here that a main goal of music theory must necessarily be to develop an idiostructural symbology is practicable, it should be emphasized again, only in the context of a real hierarchical theory. A onedimensional, one-level setting of an idiostructural symbology would defeat itself in a maze of notational density. Indeed, though we can be sure that a symbological analysis of the idiosyncrasies in an artwork would generate exceptionally complex representations, it is only with the emergence of hierarchical theory that the concept of an idiostructural symbology even becomes feasible. For hierarchies, as I have discussed, enable one to analyze complexities without falling prey to the trap of an inadequate, distorting, and misleading reductionism.

One possibility for insuring the legibility of an idiostructural symbology in a field like music would be to divide each hierarchical level into stages of closure and nonclosure for each event. Each of these stages would then be divided further into each operating musical parameter (melody, duration, harmony, etc.). Each parameter could then be viewed still further in terms of inborn cognitive constraints and learned variables since both the "personal and public" sense of style would have to be taken into account. Finally, style would have to be incorporated according to the impingements of endogenous (intraopus, intracontextual) and exogenous (interopus, intercontextual) factors. In other words, in order to generate a comprehensive 
idiostructural symbology, one would move more and more "downward" toward defining hierarchical properties within a given level before moving "upward" toward any transformation.

Hence, any idiostructural analysis representing such a "divide-and-conquer" program would resemble not so much a reduction as a translation. ${ }^{22}$ And though at this stage we can only begin to contemplate what an idiostructural analysis might look like-since the concept of true hierarchy, as this article argues, is just now beginning to be understood-the development of an analytical symbology with such capabilities must, I believe, be a major goal for music theory. Indeed, it can be argued that idiostructural understanding is a major rationale for the existence of all fields of humanistic inquiry-the raison d'être for their disciplinary status in the world of knowledge.

Finally, and epistemologically, it should be pointed out that an idiostructural symbology would offer the possibility of making formal logical arguments in humanistic fields like music theory. For hierarchical idiostructural analyses will generate exceptionally complete analytical reductions-or rather translations-ones in which the terms of the theoretical statements would become hard definitions in the analytical theorems. This in turn would allow for the first time the construction of powerfully consistent musical theories incorporating internally a minimum of rationalistic belief with all that entails for revitalizing the humanities as essential intellectual disciplines in higher education.

\section{The Complexity of Musical Hierarchies: Tree Progressions and Horizontal Networks as Simultaneous Aspects}

It is clear, however, that we can never really construct anything remotely resembling a style-structural or an idiostructural symbology unless we attempt to generate theoretically and analytically "real" hierarchical transformations as opposed to style-formal systemic ones. The central desideratum in this respect is to preserve partial decomposability. To comprehend precisely how the constituents of a given level are both self-contained and yet simultaneously other-dependent is the key. Put in psychological terms, we must define how a level can function internally as both open and closed at the same time. For in a true hierarchy, a given level must be analyzed and symbolized in partially nonreducible terms with respect to the level itself rather than just in terms of the endogenous relationships established either later in time or at higher levels or in terms of the exogenous relationships existing prior to the level in question.

22. For a tentative, imperfect example, see the analysis of the theme from the last movement of Beethoven's Sixth Symphony in my paper (Narmour, in press). 
Such partial independence of levels is, as we have seen, what distinguishes a hierarchical approach from a systemic one. ${ }^{23}$ However, because of the syntactic properties of music, the kind of partial decomposability inherent in temporal hierarchical levels tends to create specialized analytical connections. For the fact that both closure and nonclosure can exist simultaneously on the same level in a piece of music greatly increases the possibility that "horizontal" networked hierarchies will occur in addition to "vertical" tree-structured ones. ${ }^{24}$

Now what distinguishes networked hierarchies from tree-structured ones is that in networks, connections between levels tend to be asymmetrical and nonsystematic. ${ }^{25}$ In music such asymmetric connections are brought about primarily in two kinds of ways. First, some nonclosural event may become closed discontiguously; that is, an implication may be denied in prospect but then become realized in retrospect later on. Second, an event that appears closed and disconnected in prospect may be "activated" later in time, creating in retrospect a syntactic connection that was not originally implied. ${ }^{26}$

It is instructive at this point to consider two different analyses of the same example. The first analysis of this humdrum, synthetic tune displays a typical tree-structural design (Figure 7). Functional relations among pitch, duration, and meter are displayed on seven different levels, levels being expressed in terms of the durations of the structural tones (sixteenth notes, eighth notes, quarter notes, half notes, etc.). ${ }^{27}$ Though it is perhaps questionable how "real" the pitch relations are on both the $3 / 2$ level and the $6 / 2$

\section{For further discussion see Narmour (1977, chapter 8).}

24. The existence of such networked possibilities, of course, vastly complicates the music theorist's job since, if all hierarchical musical relationships were treelike, it would be much simpler to analyze and explain musical phenomena. On the other hand, if this were so, it is unlikely that music would yield the same quantity and quality of aesthetic effect since the continuing regeneration of aesthetic delight, even after countless rehearings, is doubtless due directly to the inherence of hierarchical complexity.

25. The relationships I have in mind resemble what computer scientists working in artificial intelligence sometimes call "heterarchical."

26. Thus, the "meaning" of any such event cannot be explained only in retrospect-by what eventually happens to it. What we need in the study of music is to discover the cognitive constants that explain perceptual expectation separately from perceptual realization-a theory of nonbinding implication.

27. In a hierarchy a tone on a higher level functions on the levels beneath it as well. Thus, for instance, the half-note $\mathrm{C}^{2}$ functions on the sixteenth-, eighth-, and quarter-note lower levels as well as on higher levels. Likewise, the C-E-G in measures 1-2 also functions on the sixteenth- and eighth-note levels, though for the sake of clarity that is not shown in the example. 

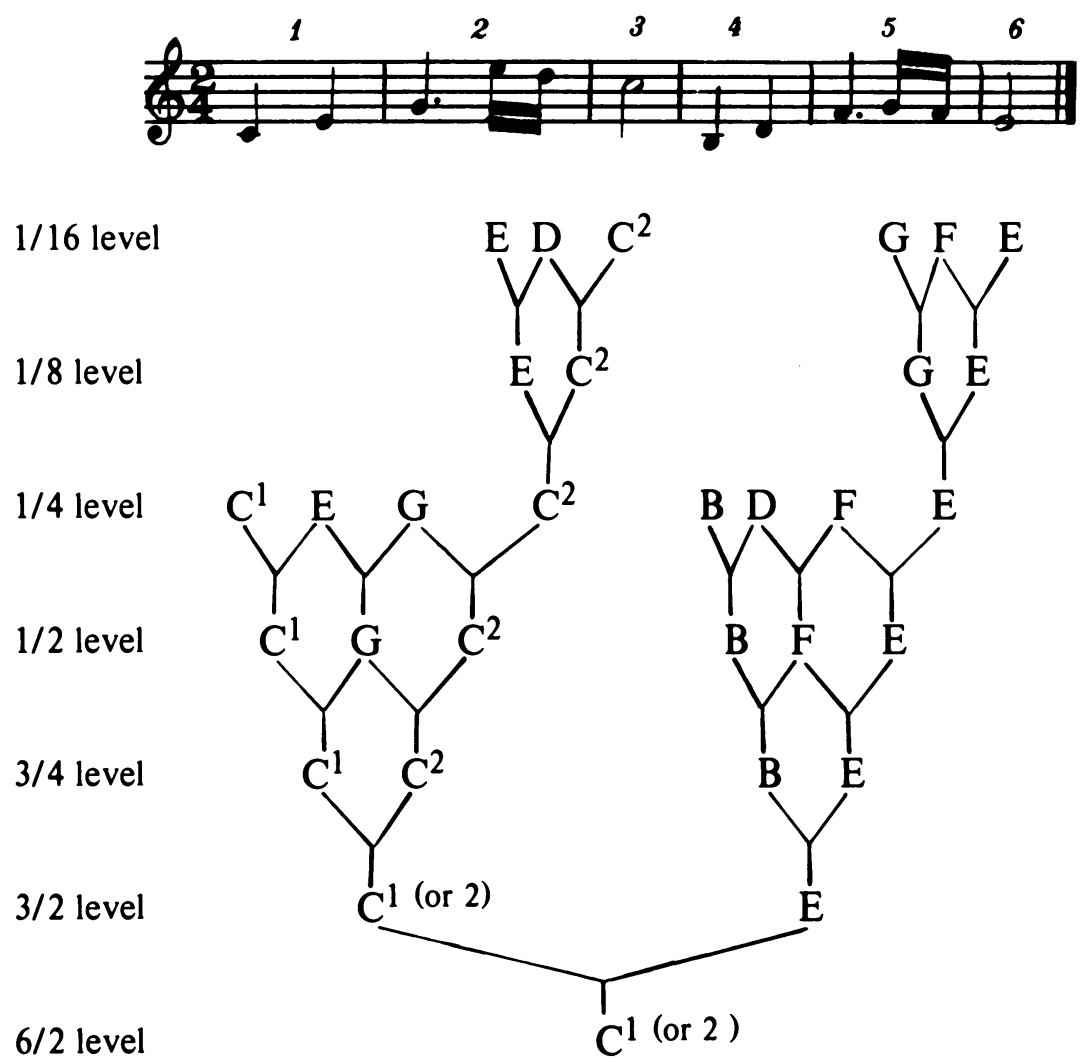

Figure 7.

level, the pitch analysis shown is perfectly derivable from the rules of several currently popular theories of tonal music. ${ }^{28}$

But immediately we see several problems. First, there is the lack of a registral connection in the analysis between the low $C$ in measure 1 and the low $B$ in measure 4 , a relationship based on the conformance between the two phrases which most listeners would assert as a perceptional-experiential fact. That is, in the repetition of phrases in Figure 7, a melodic, rhythmic, and formal similarity $\left(\mathrm{A}^{0}+\mathrm{A}^{1}\right)$ occurs between measures $1-3$ and $4-6$, connecting the initial $\mathrm{C}$ of measure 1 to the $\mathrm{B}$ starting the second phrase in measure 4 (see Figure 8). In this respect, the tree-structured analysis thus

28. For example, Leonard Meyer's theory of choosing structural tones via levels of metric coincidence or Schenker's theory of voice-leading reductions (excepting the $6 / 2$ level). I shall discuss both of these theories shortly. 


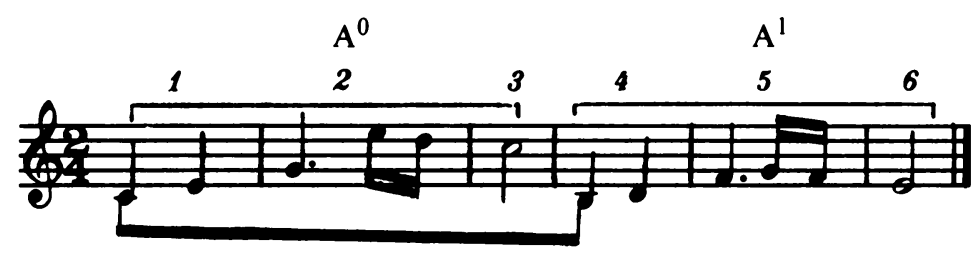

Figure 8 .

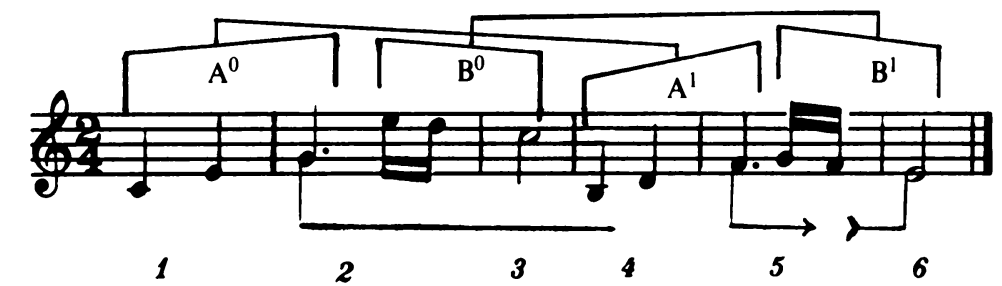

Figure 9.

appears incomplete since that relation does not show up. Indeed, one cannot make that relationship analytically anywhere in the tree diagram: traveling through the various levels of Figure 7 yields no direct, contiguous levelconnection between the low $\mathrm{C}$ and the low $\mathrm{B}$.

Nor are these two phrase-initiating notes the only surface-level discontiguous relations to occur that are omitted in the tree analysis. Because of conformant similarity $\left(A^{0}+A^{1}\right)$, for instance, a connection between the $G$ in measure 2 and the first $\mathrm{F}$ in measure 5 takes place as well on account of the fact that both of these pitches terminate rising melodic triads (C-E-G, B-D-F), that both cumulate durationally (on dotted quarters), and that both pitches are registrally proximate (a whole step apart). In short, these melodic and rhythmic functions closurally draw attention to the G-F relationship, ensuring the discontiguous connection between the two notes on the half-note level and creating a descending line that goes on to the $\mathrm{E}$ in measure 6 (see Figure 9). ${ }^{29}$

29. Note as well in typical fashion that the horizontal networked line between the discontiguous $\mathrm{G}-\mathrm{F}$ and $\mathrm{E}$ creates a modicum of asymmetry in what otherwise appears to be a patent $3+3$ phrase: The $G$ to the $F$ is spaced discontiguously by five intervening tones and two and one-quarter intervening measures, whereas the $F$ to the $E$ is separated only by two sixteenth notes (G-F, measure 5). For these reasons we thus experience a sense of structural acceleration when the $\mathrm{F}$ moves to the $\mathrm{E}$. 
For the same reasons of conformant similarity, we relate the E-D-C of measures $2-3$ to the discontiguous $\mathrm{G}-\mathrm{F}-\mathrm{E}$ of measures $5-6\left(\mathrm{~B}^{0}+\mathrm{B}^{\mathbf{1}}\right)$, despite the fact that the initiating sixteenth-note $E$ of measure 2 is preceded by a leap of a sixth in contrast to the initiating sixteenth-note $G$ in measure 5 which is preceded by a mere step below. And though the pitch disproximity between these two motives is clearly such that one would not feel comfortable joining the initiating pitch $\mathrm{E}$ of the $\mathrm{B}^{0}$-motive (measure 2 ) to the initiating $\mathrm{G}$ of the $\mathrm{B}^{1}$-motive (measure 5 ) or the terminal $\mathrm{C}^{2}$ of measure 3 to the terminal $\mathrm{E}$ of measure 6, both pairs of motives are nevertheless linked both formally and functionally-overlaying a horizontal network of pitch connections on top of whatever underlying vertical tree-design there is.

One flaw, then, in tree-structured hierarchies is that important relationships are often simply ignored. Of course, if this incompleteness only affected discontiguous conformant relations, as in the case of the low $\mathrm{C}$ to the low $\mathrm{B}$, then this problem might be easily correctable. However, as we shall see, tree-structured analyses also generate anomalies with respect to contiguous relations. ${ }^{30}$

For instance, in the tree analysis of this tune (Figure 7), there is a gaping hole between the high $\mathrm{C}\left(\mathrm{C}^{2}\right)$ of measure 3 and the immediately contiguous low $B$ in measure 4. Between the first phrase (measures 1-3) and the second (4-6), the analysis creates a disjunction which cuts through all the levels before the low $B$ vanishes altogether. The downward leap of the minor ninth is simply omitted from the tree scheme, as if it were a nonexistent interval. Explanatorily, it is an intervallic "default" instead of the "dramatic" descending jump from which the second phrase is initiated. One supposes that the leap from $\mathrm{C}^{2}$ down to $\mathrm{B}$ can be included on some level in the tree diagram, and indeed it can, but, as can be seen in example (a) of the following diagram, it will tend to create other kinds of analytical difficulties. If, for instance, we try to accommodate the $\mathrm{C}^{2}-\mathrm{B}$ interval on a relatively high level, then the $\mathrm{C}^{2}$ in measure 3 must "drop out" of the analysis earlier than it does in example (b), becoming permanently downgraded to the relatively less significant $3 / 4$ level, an untenable conclusion in the face of our perceptual experience of the note closing off the first phrase. If on the other hand we try to maintain the $\mathrm{C}^{2}$ at a higher level and yet make the $\mathrm{C}^{2}-\mathrm{B}$ connection at a lower level, example (b), then the low $\mathrm{B}$ initiating the second phrase disappears after the 3/4 level-an equally unacceptable solution for the same formal reason. 


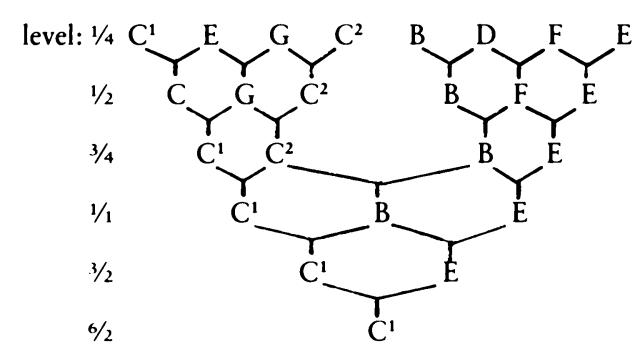

(3)

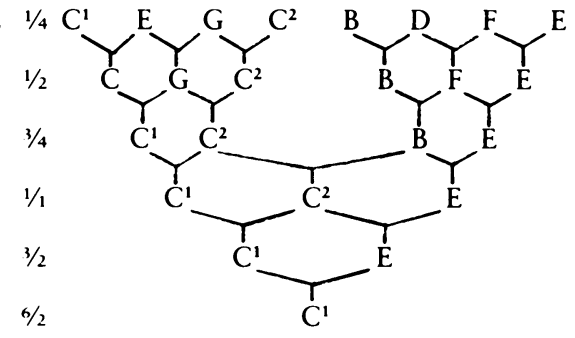

(b)

More objectionable, however, is that in trying to make a connection between the $\mathrm{C}^{2}$ and the low $\mathrm{B}$ in the context of a tree analysis, we create a false level at the duration of the whole note (1/1). For in no way are the pitch structures at the $1 / 1$ level $\left(\mathrm{C}-\mathrm{B}-\mathrm{E}\right.$ in $[\mathrm{a}]$; $\mathrm{C}-\mathrm{C}^{2}-\mathrm{E}$ in $\left.[\mathrm{b}]\right)$ tenable. The sixmeasure melody is simply not structured around a three-note sequence. The six bars are not divided into three two-measure phrases $(3 \times 2=6)$, but rather into two three-measure phrases $(2 \times 3=6)$, each resting on a pair of structural tones as shown on the $3 / 4$ level.

The distorting, rationalistic $1 / 1$ level results, of course, from analytically superimposing a relentless symmetrical tree-structured "vertical" form onto what is essentially a temporal "horizontal" phenomenon. This is not to say that the tree analysis of the structure of the melody in Figure 7 is totally erroneous; as we saw earlier, the level-progression of structural tones does demonstrate why this little tune is perceived as two three-bar phrases. But on the negative side, the tree parsing has considerable difficulty accounting for the manifestly contiguous $\mathrm{C}^{2}-\mathrm{B}$ melodic relationship between measures 3 and 4 . Such typical asymmetrical cases raise empirical questions as to the appropriateness of tree-strucutral diagrams in music analysis. Indeed, it is a characteristic flaw of tree-structured generations in music analysis that manifestly contiguous relationships constantly get omitted.

To summarize, analysis by tree levels tends to ignore relationships that are not systematically congenial to the symmetric progression of the diagram. The omission of both manifestly contiguous and discontiguous relationships is common. And though a tree analysis can attempt to incorporate all the perceived connections, it usually does so at the expense of generating false, rationalistic, nonempirical levels with all that means for distorting actual hierarchical functions. To be sure, neither the tree analysis by itself (Figure 7) nor the network analyses by themselves (Figures 8 and 9) are sufficient to generate all of the contiguous and discontiguous pitch relationships that occur. In order to explain the pitch relationships of our little synthetic melody, the horizontal network would have to be mapped onto the vertical tree, including in the analysis all the manifest relations on every level such as to ensure the integrating of both stylistic and idiosyncratic 


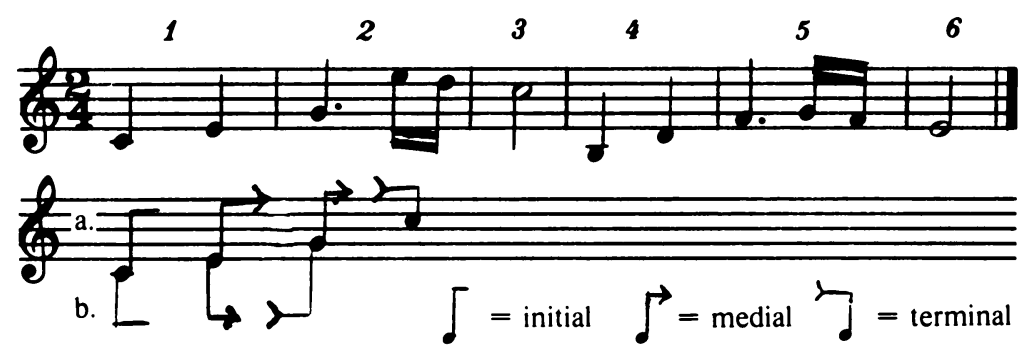

Figure 10.

aspects. In short, musical analysis must admit the possibility of the simultaneous existence of both treelike and networked level-relationships.

This possibility has, as it turns out, curious ramifications as regards the concept of functionalism and time in musical hierarchies. Consider the $G$ in measure 2 of our synthetic example, for instance. We saw in the tree-analysis (Figure 7) how it functioned on the quarter-note level as the medial part of an ascending triad to the high $C^{2}\left(C-E-G-C^{2}\right.$, measures 1-3). At the same time we saw in the network analysis how it functioned on the quarter-note level to initiate the discontiguous, descending G-F-E line (Figure 9). Finally, we observed that it also functioned durationally to terminate the triad CE-G, thereby creating a form whose conformant similarity was recognized with the B-D-F of measures 4-5. Thus, this same G functioned initially, medially, and terminally. But how can such antithetically contrasting analytical functions on the same note occur without generating extreme melodic ambiguity?

The answer has to do with the successive transformations this pitch undergoes over the course of time. When it first appears, we surmise the formation of a C-E-G triad that implies an octave realization on to the $\mathrm{C}^{2}$ (see Figure 10a). As time goes on in measure 2, however, and the $\mathrm{G}$ becomes prolonged to the length of a dotted quarter, we reevaluate our previous assessment and, in view of the durational closure, come to feel that $G$ is to be a structural tone, a transformation closing the C-E-G ascent thereby denying the high $\mathrm{C}$ (Figure 10b). Even up to the last half-beat of measure 2 this reevaluation appears correct inasmuch as a leap up to the sixteenth note followed by a reversal on D occurs. But immediately the previously implied octave now becomes realized after all on the ensuing $C^{2}$. Thus, the earlier transformed $G$ now functions medially; that is to say, as $C-E-G-C^{2}$ is realized in retrospect, its prior function as a structural tone is deformed. Yet this is not the end of the matter. Two measures later in retrospect, the $G$ earns once again structural status via the conformant similarity, initiating the networked, discontiguous descending line with the F of measure 5 , as we saw earlier in Figure 9.

Thus, we are forced to reevaluate our perceptions and revise our analysis 
back and forth-from a function of formation to transformation to deformation to transformation-in order to comprehend the complex functional meaning of the $G$ in measure 2 . It is in fact just these kinds of fluctuating transient functional changes that create the highly reticulated vertical and horizontal hierarchical complexes found in music-causing cohesion between the internal parts of artworks and making possible the variety of our aesthetic experiences. It is also this complexity that makes the formulation of music theory exceptionally difficult. Even if we understood and properly applied the concept of hierarchy, there remains the considerable perplexity of how we are accurately to sort out such multivalent network functions. Our synthetic example is about as simplistic as we can get, yet even here pitch relationships are quite complicated. We can hardly imagine how much more dense hierarchical complexities are in real pieces of music-where many more parameters other than melody and duration are operating.

\section{On the Nature of Partially Decomposable Levels in Music}

It will be profitable for our purposes to inquire further into the nature of decomposable levels in music. Given that both vertical tree-structures and horizontal networks seem necessary in the hierarchical conception of musical levels, how would partially decomposable levels come about? I have already indicated the answer: by the emergence of partial closure. But if this is true, it raises the question of measuring, or at least evaluating, the degree of closure that occurs. For if incomplete closure is a typical feature of musical hierarchies, then what amount of closure would suffice to create the emergence of a hierarchical level? Or, seen from the aspect of nonclosure, what would sufficiently deform an event of closure such that no transformation, no emergence of a level, would occur?

The complicated fact in music analysis that hierarchical levels may possess only partial closure has peculiar ramifications. Since levels also generate forms, the forms of musical hierarchies themselves may thus be only partly complete, only partly closed, in and of themselves. And in a true hierarchy (as opposed to a system), such formal incompleteness representing a level must be temporally defined not just in terms of either an earlier or a later transform but also in terms of the formal dynamics of the given transform itself. And since such formal partialness would depend not only on some degree of internal closure but also on the presence of internal nonclosure, it follows that in a musical hierarchy all forms, that is, all levels, must be conceived as potentially both closed and unclosed simultaneously in and of themselves. As we have seen, it is this strange duality that bonds musical levels (musical forms) into unified reticulated hierarchical complexes combining both tree and network effects. Of course, in any given form closure and nonclosure rarely coexist in the same degree. If closure is strong enough, 
a transform-and concomitantly a new level-will occur. If nonclosure dominates, then neither transform nor a new level will take place. ${ }^{31}$

As I have mentioned, music theorists have traditionally conceived of levels as being systemic rather than truly hierarchical, probably because they have concentrated on the analysis of style forms instead of on the critical analysis of style structure and idiostructure. Consequently, closure has been viewed as a by-product resulting from the generation of levels via a "standard transformational model": a dictionary of presumably known transforms is brought to bear on the surface structure of a piece, stamping out the forms systematically and arranging certain elements recursively into homologous transformations at ever-higher levels. In this standard model of analysis, closure is thus determined in advance by the prior collection of the transforms, and the dialectic between closure and nonclosure within any given form becomes trivial and irrelevant. This approach has two major defects in musical analysis. It tends to generate tree-structural levels whereby many contiguous and discontiguous relations, like the ones we discussed earlier, are omitted. And it perforce relies on dominating, assimilating, summarizing, and rationalistic, a priori principles to put together the dictionary of transforms so as to make them analytically operational. ${ }^{32}$

In a true hierarchical analysis, however, closure cannot be treated as a byproduct of the existence of prior transforms but rather must be regarded as the central issue governing the very emergence of levels since degree of closure is the crucial factor by which hierarchical levels come into being or are denied existence. Thus, if what is desired is a truly hierarchical conception of analysis, we must come to grips with this matter of closure and nonclosure. But where are we to find the rules governing closure and its symmetrical counterpart, nonclosure, in an analytical theory of music?

Ultimately, of course, the principles governing closure and nonclosure can only be discovered in the perceptions, cognitions, and learning habits of the music listener. But since the principles governing these things as they apply to the complexities of musical artworks are at present only vaguely understood in psychology and psychophysics, we must look for the interplay between the two fundamental factors of closure and nonclosure in the individual parameters of music itself. ${ }^{33}$

31. In the latter case, the weak degree of closure will make its appearance as a low-level articulation.

32. See Narmour (1977, chapter 9) for an extended discussion of this approach and its flaws with respect to music.

33. By parameter I refer to melody, harmony, duration, etc.-in a word, all those syntactic properties of music that can be scaled, following the meaning of the Greek roots making up the word (para = alongside of; meter $=$ measure). 
For since all the parameters of music-melody, duration, harmony, texture, timbre, tessitura, tempo, meter, register, dynamic, etc.- contribute toward the emergence of musical forms, we must therefore allow for the possibility that each of these parameters also contributes its own closural and nonclosural effects to the analysis at hand. That is, in the production of hierarchical levels, each parameter must be regarded as an independent component operating under its own closural and nonclosural rules. To create a truly hierarchical theory, then, would mean first discovering the internal closural and nonclosural rules for each parameter and then devising integrative rules for determining the degree of overall closure necessary for the emergence of levels. This is a tall order indeed, which may explain why most music theorists continue to be attracted to less complex analytical theories employing some set of standard transformations for generating systemic levels.

Merely isolating these independent parameters has yet to be done with any rigor perhaps because musical relationships enter our cognitive apparatuses as extremely integrated complexities whose networked cohesiveness embodied in an idiostructural and style-structural hierarchy makes objective analysis acutely difficult. It is very hard, for instance, to imagine pure melodic relationships without also involving duration or harmonic relations in the guise of voice-leading. Likewise, the elementary design of any single durational pattern automatically evokes the particular style it comes from with surprising accuracy. Even the most abstract patterns we can imagine trigger a panoply of learned stylistic evocations. All kinds of relationism thus invade our efforts to conjure up and externalize the parameters of music. ${ }^{34}$ And until we are able to define them much more precisely, severely restricting the amount of contextual interpretation that must be invoked in order to make consistent the use of our terminological concepts, we shall continue to have difficulty formulating the closural and nonclosural rules governing parameters, consequently delaying the discovery of a defensible theory that will generate true hierarchical levels.

34. Perhaps the clearest evidence of such difficulties is to be found in our theoretical terminology, a quagmire of concepts the murky likes of which epistemology has rarely encountered. Take the term "rhythm," for instance. It is often used in a summarizing sense to refer to the totality of musical relations present in all the parameters at any given moment. Sometimes we employ the term to refer to similarities of pitch contour in a melody, that is to say, to describe a form. Other times we routinely use it to describe tempo (e.g., "fast rhythms") or to refer to what is in fact meter-as, for example, in saying that a phrase is "in the rhythm of $2 / 4$." Sometimes we use the word strictly to refer to durational patterning. Indeed, no term (with the possible exception of texture) is used more inconsistently in music analysis than the word "rhythm." 


\section{Some Parametric Hypotheses}

By way of illustration, however, let me attempt to define certain aspects of closure and nonclosure with reference to the primary parameters of music. I have suggested why each parameter must be imagined as possessing its own closural and nonclosural rules of operation and why the emergence of partially decomposable, hierarchical levels is dependent on the way parameters interact in the production of higher-level transforms. And though, like any other analytic field dealing with artifactual phenomena, the rules of perception and cognition are bound to involve both inborn (innate) and learned operations, making theory construction all the more difficult and besetting us with still more perplexities in our quest to discover empirically defensible hierarchical levels, there are nevertheless certain concepts-certain things we think we know for sure-that we can call upon as hypotheses in our search for hierarchical levels. Let us investigate a few of these concepts and apply them analytically to a common example as a way of illuminating what has been said about the major problems of analyzing musical hierarchies so far.

We will restrict ourselves to three primary parameters: melody, harmony, and durational patterning (avoiding the word rhythm altogether). By melody, I mean simply the horizontal succession of pitches-with no reference to tonic or dominant or the diatonic scale or any other contrivance of tonality. By harmony, I mean the succession of intervallic verticalities without any reference to voice leading. And by durational patterning, I mean just the actual durations appearing on the score page, with no reference to the meter, barline, or accent.

Now with respect to harmony, as here conceived, the concept of closure seems fairly well defined: harmonic closure can be said to occur either when dissonant intervals (seconds, sevenths, tritones) move to consonant ones (unisons, octaves, fifths, fourths, thirds, sixths) or when chords in inversion (e.g., with the third or the fifth in the bass) move to chords in root position (with the fundamental of the chord being the lowest note in the actual bassline). ${ }^{35}$

With respect to the parameter of melody, however, the concept of closure is on shakier grounds. I will hypothesize the following principles as determining melodic closure:

a) Registral return, exact or nearly exact. An example of exact return would be a neighboring tone, for example, the pitch sequence $\mathrm{C}-\mathrm{D}-\mathrm{C}$ or any other motion where the initial pitch is returned to (e.g., C-E-C, C-F-C, C-G-C, etc.); an ex-

35. The position of the soprano is obviously also of fundamental importance with respect to closure, but I will not deal with that here. 
ample of near-return would be the recurrence of a pitch that is within a half or whole step of the original one (e.g., C-D$\mathrm{B}$, or C-D-Bb).

b) The termination of a pattern of registral continuation, where continuation is defined as two or more like intervals in the same registral direction, the difference between them being no more than one whole step (e.g., the ascending patterns of C-D-Eb, C-D-E, C-D-F, C-D-F\# exemplify continuation whereas an ascending pattern of C-D-G would exemplify noncontinuation). Termination may occur either by reversal of registral direction or by the simple stopping of the pattern (e.g., in the ascending-descending pattern of $\mathrm{C}-$ $\mathrm{D}-\mathrm{E}-\mathrm{D}-\mathrm{C}$, a reversal occurs on the $\mathrm{E}$; in the ascending pattern of $\mathrm{C}-\mathrm{D}-\mathrm{E}-\mathrm{A}-\mathrm{B}$, a termination of continuation, as defined, would also be said to occur on the E).

Correlative to this latter rule, I will also hypothesize a rule of primacy for the identification of hierarchical levels:

c) The initiation of a pattern of registral continuation (e.g., in an ascending pattern of $\mathrm{C}-\mathrm{D}-\mathrm{E}$ initiation occurs on the C). ${ }^{36}$

With respect to the definition of the parameter of durational patterning, we are also somewhat at a loss as to a concept of closure, but for the purposes of discussion I will propose the following simple rule: any duration that moves cumulatively to another duration will be said to be closural (e.g., $\delta d$ or any other given time-value that moves to a longer time-value). Conversely, any pattern that moves countercumulatively (e.g., d $d$ ) will be hypothesized as nonclosural. This leaves only the class of additive durations (e.g., d ) which may be hypothesized as weakly nonclosural patterns of attenuated continuation. ${ }^{37}$

\section{Parametric Applications: Harmony and Duration}

Thus armed with these hypothetical analytical princples, which can be applied recursively on successively higher levels, let us see how their appli-

36. Strictly speaking, initiation, of course, does not exemplify closure in a syntactic system but rather belongs to a larger class of structuring operations. But for the sake of economy in this paper, I will lump initiation and termination together under the closural rubric.

37. I have borrowed the concepts of additive, cumulative, and countercumulative durational patterns from Leonard B. Meyer. It should be pointed out that, though the durational patterning of any one melodic line is ordinarily represented fairly well in the actual notation on the printed page, the durational patterning of the harmony may not be manifestly so symbolized. 

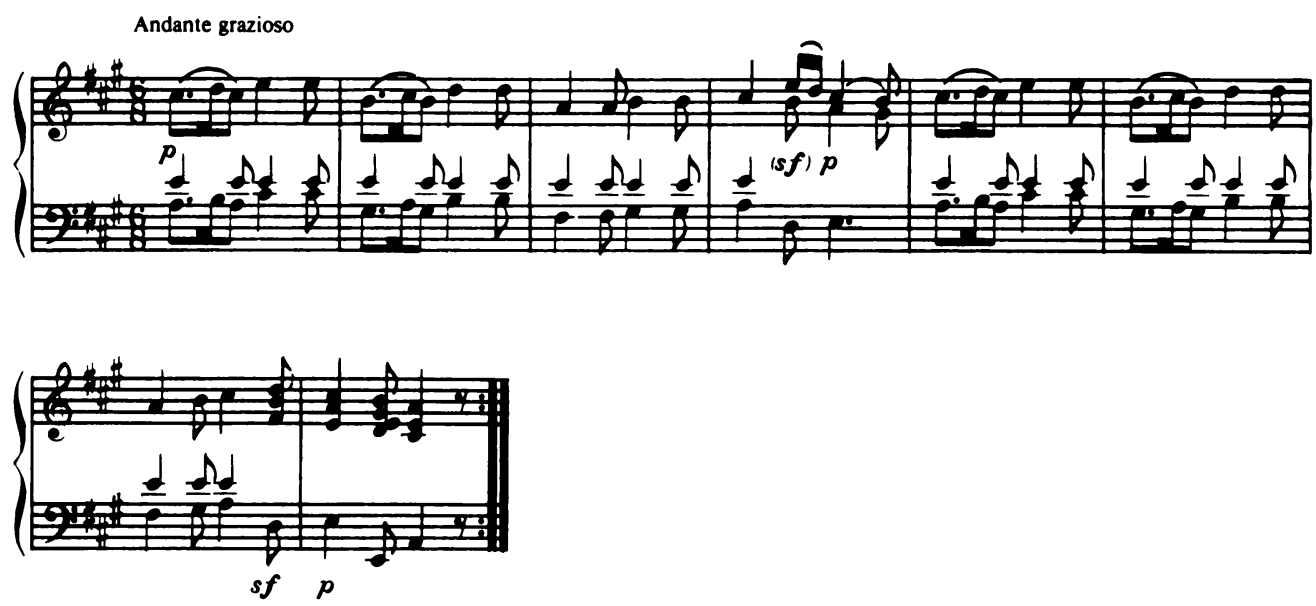

Figure 11. Mozart, Sonata for Piano, K. 331, I, measures 1-8.

cation sheds light on the problem of generating levels of transformations. For purposes of comparison, we will examine a subphrase of the theme from Mozart's Piano Sonata in A Major (K. 331, Figure 11), one of the most overanalyzed pieces in the history of music theory-and one of the most "tree-structured" themes known-but for these reasons all the more relevant for the sake of our discussion.

In the analysis of the note-to-note level in Figure 12, the points of closure (and primacy) in the parameters of melody, harmony, and duration (as previously defined) are symbolized by the letters CL. ${ }^{38}$ Reading vertically via the dotted lines, one can see how the separate parameters interact creating congruences of varying closural strengths. Toward the bottom of the analysis, the number of closures for each pitch of the phrase is tallied. In general, the higher the number, the more closed the pitch; and the more closed the pitch, the more strongly it transforms to the next level-though the sums shown are very rough since the degree of closure in any given parameter varies from note to note and since the analysis does not take into account next-level interactions of closure. Moreover, because in several cases the nonclosure present in one or more parameters is actually stronger than the closure, the count, as we shall see, may be of little significance as regards the emergence of levels. Obviously, any theory measuring degree of closure in a piece of music would require a good deal more statistical sophistication than the approximations shown here.

38. By inference, of course, the absence of a $\mathrm{CL}$ in following the dotted lines from parameter to parameter indicates the presence of nonclosure. 


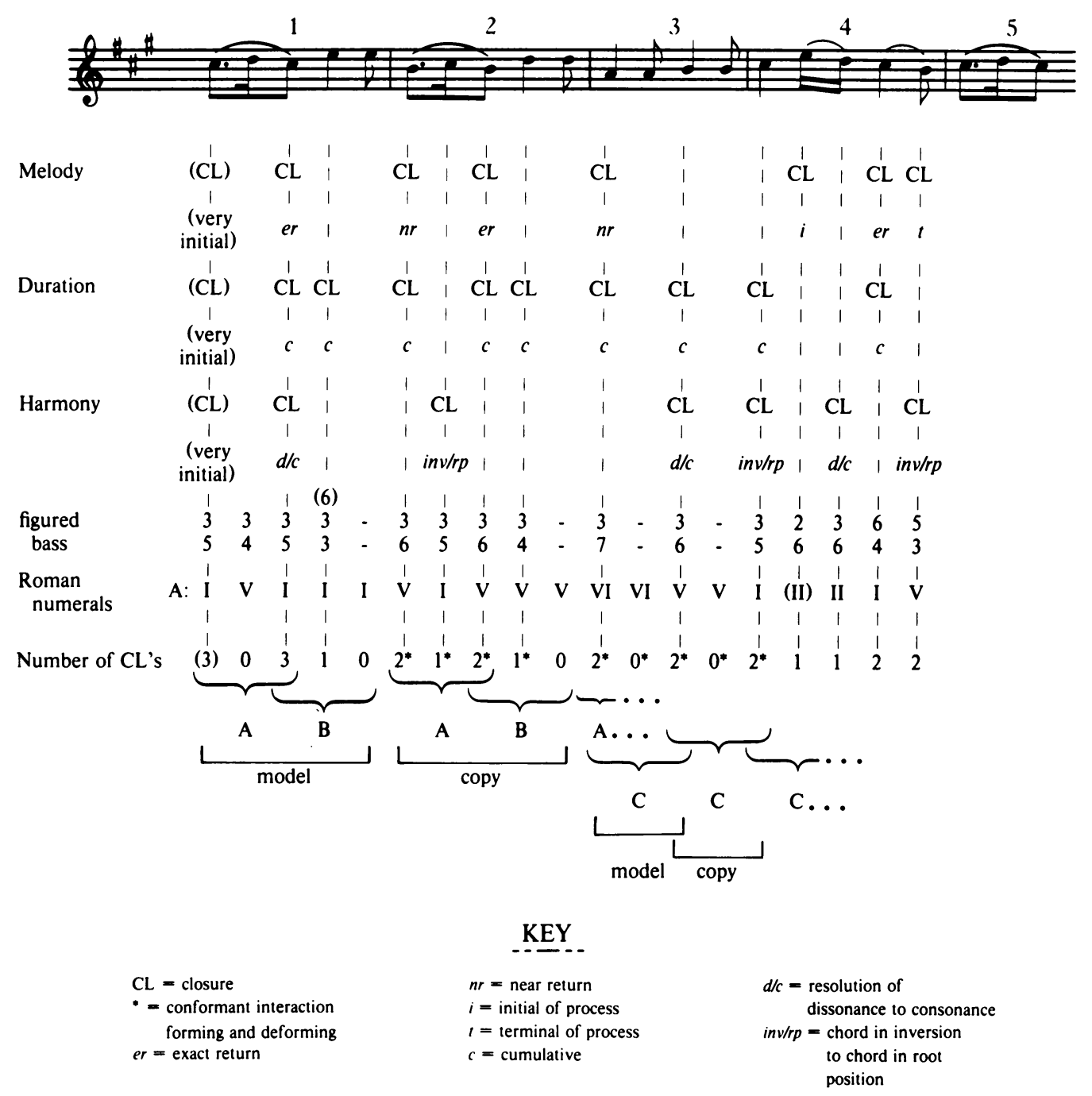

Figure 12.

Let us now briefly examine the analysis of each parameter. The least complicated is that of duration. The points of cumulation can be easily traced from left to right. With one notable exception, the durational patterning in this piece is remarkably uniform. The sixteenth note to the eighth note on the second $\mathrm{C} \#$ in measure 1 and on the analogous second $\mathrm{B}$ in measure 2 has a cumulative ratio of $1: 2$ as does the eighth-note $C$ to the quarter-note $\mathrm{E}$ in measure 1 and the eighth-note $\mathrm{E}$ to the quarter-note $\mathrm{B}$ in measure 2. Indeed, this lilting durational pattern dominates throughout the 
phrase except for measure 4 where, as the sixteenth-notes E-D move to the quarter-note $C \sharp$, durational closure is a measurably greater event, creating between the $\mathrm{D}$ and the $\mathrm{C} \#$ on the second beat a ratio of 1:4.

Obversely, the same kinds of observations could be made about the varying degrees of durational nonclosure: the initial dotted-eighth $\mathrm{C}$ to sixteenth-note $\mathrm{D}$ in measure 1 and the parallel B-C\# in measure 2 countercumulate nonclosurally in ratios of $3: 1$. All other countercumulative durational patterns are 2:1 (the $d \delta$ in every bar) with the exception again of measure 4 where the quarter-note $\mathrm{C}$ going to the sixteenth $\mathrm{E}$ moves more strongly toward nonclosure at the ratio of $4: 1$.

Let us next consider the parameter of harmony. Since here we begin to encounter noncongruence-that is, relationships where nonclosure in harmony competes with closure in duration - we will be forced to make decisions as to which parameter "dominates," either creating or preventing the emergence of levels. One theoretical advantage of analyzing parameters separately is that it forces us to formulate and test for consistency our analytical hypotheses about such noncongruent interactions.

For the first three notes of measure 1 , harmonic and durational closure is completely congruent as Figure 12 shows. The sixteenth-note $\mathrm{D}$ is both dissonant (a seventh over the E of the middle voice; see Figure 11) and countercumulative; and this congruent nonclosure resolves (i.e., closes satisfactorily) on the return of the consonant (symbolized $d / c$ ) cumulative $(c)$ eighth-note $\mathrm{C} \# .^{39}$ With the quarter-note $\mathrm{E}$ in measure 1 , duration is closed (cumulative) while harmony, as a movement from a root-position chord (root in the bass) to a first-inversion chord (third in the bass), is open. Duration with respect to the transformation of this $\mathrm{E}$ thus acts as an instrument of formation while harmony functions as an instrument of deformation.

Can we make a case on this $\mathrm{E}$ for one parameter dominating over the other? Or do they cancel each other out, so to speak? It seems reasonable to assert that in this instance duration clearly dominates. For a harmonic motion in which the fundamental between the two chords remains the same is clearly a very weak progression. And for that matter, a root-position chord moving to a first-inversion one ( $\mathrm{I}-\mathrm{I}_{3}^{6}$ in Roman numerals) is not a strong progression either, whereas a cumulative durational pattern where the second note is twice as long as the first would appear to be a relatively strong temporal motion. Thus, it seems safe to say the quarter-note $\mathrm{E}$ in measure 1 makes a strong bid to move to a new level. From this, we can

39. The very first $\mathrm{C}$ of the piece is tagged as closural in the parameters of melody, harmony, and duration since it initiates the phrase and is marked in the mind as a signal event of primacy. 
extrapolate a specific hypothetical rule of analysis: Whenever noncongruence between harmony and duration exists and harmonic motion is from a root-position chord to a chord of first inversion with both chords having the same fundamental, durational closure will transform the harmonically nonclosural pitch if the closural cumulation in the pattern is at least twice that of the initial duration. ${ }^{40}$

Measure 2 affords the opportunity to formulate a similar hypothesis, though with opposite results. For with the sixteenth-note $\mathrm{C}$ we again have noncongruence between duration and harmony, except here duration is nonclosural (a countercumulative pattern of $3: 1$ ) while harmony is closural (a first-inversion chord moving to a root-position one with different fundamentals between them, $\mathrm{V}_{3}^{6}-\mathrm{I}$ in Roman numerals-symbolized as inv/rp in the example). If we are consistent, this difference in function between harmony and duration should again result in the dominance of duration (which is here countercumulative and nonclosural) since if a root-position chord to a first-inversion one is weak nonclosure, as we say in the $\mathrm{I}-\mathrm{I}_{3}^{6}$ analysis on the $\mathrm{E}$ of measure 1, then a first-inversion chord to a root-position one ( $\left.\mathrm{V}_{3}^{6}-\mathrm{I}\right)$ must also be weak closure. And though it is true in measure 2 that different roots are involved between the two chords ( $\mathrm{E}$ to $\mathrm{A}$ ), it is also true here that the durational countercumulation of the $C \#$ is a strong $3: 1(\curvearrowright$. $/)$, in contrast to the $1: 2$ closural cumulation on the $\mathrm{E}$ in measure 1 , thus offsetting-it seems hypothetically justified to assume-whatever added degree of closure the root difference in measure 2 makes. In other words, the nonclosural durational countercumulation on the sixteenth note so deforms the relatively weak closure of the harmony $\left(\mathrm{V}_{3}^{6}-\mathrm{I}\right)$ that the melodic $\mathrm{C}$ in measure 2 remains on the foreground, note-to-note level.

\section{Conformance as a Closural and Nonclosural Factor}

At this juncture in the discussion, it is relevant to discuss one other important factor to figure in the analytical evaluation of the primary parameters. And that is the phenomenon of conformance, which is to say, style. ${ }^{41}$ To be sure, any given form on any level, from tiny motives to macroforms of enormous dimensions, is a direct and demonstrable consequence of the closure brought about by the interaction of parametric congruence. At the same time, the recognition of modeling, of stylistic recurrence, of formal similarity, of repetition-in a word, of conformance-is an independent mechanism of our comprehension that is always operative regardless of

40. Provided, of course, the intervals in the parameter of melody are like the ones here. $3)$.

41. For a discussion of the importance of conformance see Meyer's book (1973, chapter 


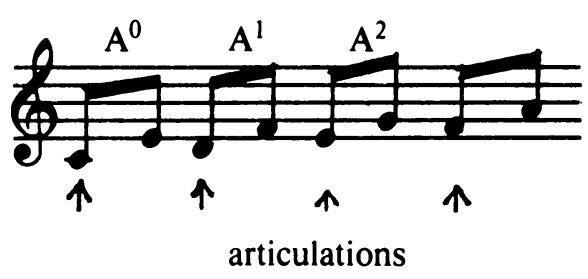

Figure 13.

whether melodic, harmonic, or durational closure-or transformation-is present. Because of this, the mere occurrence of sameness or similarity (conformance) between events will always generate some degree of closure.

The emergence of articulation in repetition is a typical musical case. In a passage like Figure 13, for instance, there is no closure present that is attributable to the melodic, harmonic, or durational rules of closure hypothesized earlier; yet undeniably we perceive the pitches in this passage as being articulated into two-note groups. That is, the nearly exact repetition causes us to "chunk" the sequence into little two-note motives because a small amount of closure in the form of articulation is created by the conformant repetition that occurs at each reversal of direction.

But conformance does not only function to create weak closure in the form of articulation. When the degree of conformance is sufficiently high, the conformance present will tend to weaken the function of the nonrecurrent elements appearing in the repetition such that they will seem to mimic functions of the original model. That is, if the given parameters of a piece of music interact closurally so as to create a memorable and thus recognizable form later on, then the recurrence of that form will not only enhance and strengthen both the closural and nonclosural functions of the copy that conforms; the recurrence will go further, attentuating the nonconforming functions that take place within the copy. ${ }^{42}$ Indeed, if overall degree of conformance is high enough, then an extraordinary assertion of nonconforming parametric independence is necessary for a nonclosural function in the first statement of the form to achieve a closural function in the recurrence, or for a closural function in the first statement to achieve a nonclosural function in the recurrence. An example of how conformance affects function will make all this clearer.

Thus, to return to our Mozart phrase, once the dotted-eighth B and the sixteenth-note $C$ of measure 2 are recognized as the beginning of a repeti-

42. For this reason, a very close analysis of the model-identifying all its distinguishing functional features-is essential in explaining music. 
tion of measure 1, conformance strengthens the closural function of the following eighth-note $\mathrm{B}$, even though it occurs over a first-inversion chord (the $V_{3}^{6}$ ), and it weakens further the preceding sixteenth-note $C^{\#}$, even though that note is part of a root-position chord (I). Put another way, the difference in harmony between the beginning of measure $2\left(V_{3}^{6}-I-V_{3}^{6}\right)$ and the beginning of measure $1\left(\mathrm{I}-\mathrm{V}_{3}^{4}-\mathrm{I}\right)$ is not enough to offset the high melodic, registral, dynamic, durational and textural conformance of measure 2 such that the function of the notes there is changed. In short, the notes at the beginning of measure 2 appear to "mimic" in function those at the beginning of measure 1 , despite the difference in the parameter of harmony.

The same could be said about the other potentially structural pitches in measure 2. The quarter-note $\mathrm{D}$, despite occurring over a dissonant chord $\left(V_{3}^{4}\right)$, appears closed because the harmonic difference from measure 2 to measure 1 is not great enough to deny the conforming influence of melody and duration and thus the formation of this D as a closural event (see the brackets and asterisks of Figure 12). (In addition to conformance, there are other melodic reasons as well why the structural tones in measure 2 mimic those of measure 1 , which shall be discussed presently.)

With measure 3 the closural role of conformance also plays a part in the $\mathrm{A}$ at the beginning of the measure even though that pitch is part of a dissonant texture (a VI occurring with a dominant pedal). That is, by conforming durationally and melodically to the sequential repetition of measures $1-2$, the quarter-note $A$ at the beginning of measure 3 implies another one-bar, intraopus, stylistic unit of like kind. ${ }^{43}$ Once we recognize at the point of the eighth-note $A$ that that is not to be, then of course the prior closure bestowed on the quarter-note A becomes somewhat weakened. ${ }^{44}$ The ensuing quarter-note $B$ of the bar on the other hand is closed on the surface level both harmonically and durationally since the dominant pedal at that point no longer functions as a dissonance..$^{45}$ The note-to-note

\section{A continuation such as}

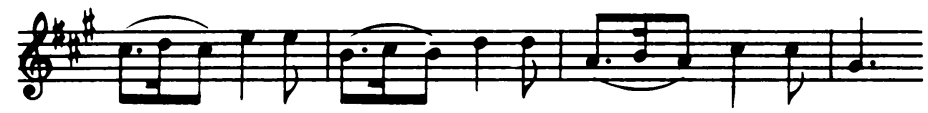

for example, would have seemed perfectly normal. I should mention, however, that extraopus norms of the style interfere with this Gestaltlike continuation. In classical music, for instance, the pattern $\mathrm{A}+\mathrm{A}+\mathrm{B}$ is so common that we are attuned toward expecting change after the beginning of the second repetition. Intra- and extraopus impingements on evaluations of closure must always be taken into account.

44. But not for long. With the quarter B of the bar, a line of ascending continuation is implied so that the $A$, as a potential initial tone, takes on the function of closure once more. The realized pattern of A-B-C\# may be seen in Figure 15, level $2 \mathrm{~b}$.

45. Formal mimicry between melodic and durational patterning during harmonic process, as we find in the Mozart example, is one of the most favored procedures in musical composition. 


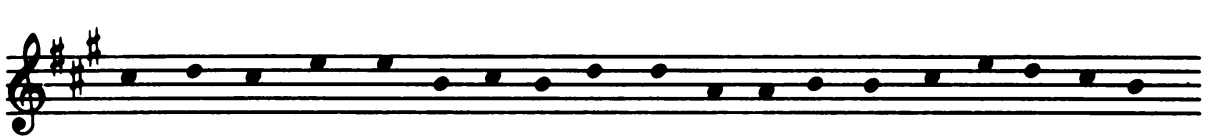

Melody

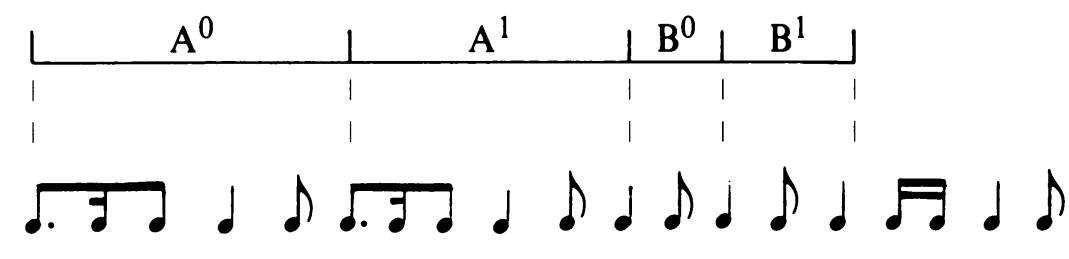

Duration:

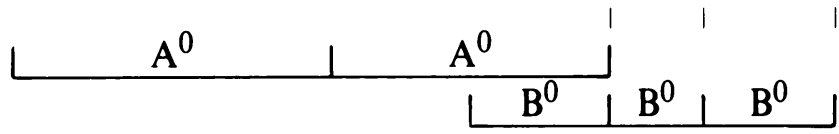

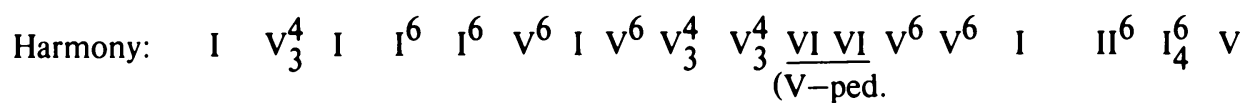
pt.)

Figure 14 .

conformant relationships are displayed parameter-by-parameter in Figure 14.

As can be seen in Figure 12, closural congruence (CL) between the parameters of harmony and duration continues to the quarter-note $\mathrm{CH}$ at the beginning of measure 4 . The sixteenth-notes E-D then break the pattern and immediately lead cumulatively $(c)$ to a $\mathrm{C}$ over a noncongruent secondinversion chord ( $\left.\mathbf{I}_{4}^{6}\right)$ while the $\mathrm{C} \#-\mathrm{B}$ motion closes harmonically on the rootposition V-chord (inv/rp). Noncongruent duration offsets this, going countercumulative and thus nonclosurally against the resolution (again see Figure 12). How the last $B$ of the bar becomes a closural, transformed tone in the face of the durational nonclosure is a function of the parameter of melody to which we may now briefly turn. ${ }^{46}$

\section{Parametric Applications: Registral Return in Melody}

One reason for the analytical popularity of Mozart's theme is doubtless that its straightforward form, harmony, durational patterning, and melody

46. Observe throughout this discussion that though I have used Roman-numeral nomenclature, it has not been necessary to rely on notions of tonality, scale step, and so forth for an understanding of the harmony. Indeed, if we were to have a satisfactory theory of music that analyzed parameters separately while at the same time formulated rules for evaluating parametric formations, deformations, and transformations, then the problem of invoking the style of tonality would become much less onerous. 
appear theoretically very unproblematic. Certainly in a melodic sense all the intervallic motions in the first phrase are relatively small-the largest skips being the perfect fourths between measures 1-2 and 2-3-so that whatever nonclosural implicative effect the intervals have is relatively weak, which prevents their performing any decisive function as a means of deformation. That is, whatever nonclosure takes place as one melodic interval moves to another is deformationally too weak to interfere significantly with the transforming closural effects of either duration or harmony or both. ${ }^{47}$

Moreover, from a registral point of view, melody functions congruently in its own special way to produce transformation of pitch and thus the emergence of levels. This is achieved on the one hand through the principle of registral return, near $(n r)$ or exact $(e r)$, as hypothesized and defined earlier, and on the other hand by the initiation $(i)$ and termination $(t)$ of patterns of continuation.

The very first motive of the piece ( $C \#-D-C \#)$, for instance, exemplifies the principle of exact return as does the analogous motive at the beginning of measure $2(\mathrm{~B}-\mathrm{C}-\mathrm{B})$. Hence, along with duration and harmony, exact melodic return in these cases contributes to the creation of closure, making the returned-to tones ( $\mathrm{C} \#-\mathrm{C}$ and $\mathrm{B}-\mathrm{B}$ ) structural on the next level (symbolized $a^{0}-b-a^{0}$; see Figure 15 ; level $\left.0, M\right)$.

Similarly, near-return of register (symbolized $\mathrm{a}^{0}-\mathrm{b}-\mathrm{a}^{1}$ ) also strengthens the transformation of melodic tones to higher levels in this phrase. The dotted eighth-note $C \sharp$ on level $2 \mathrm{a}$ in measure 1 connects to the dotted quarter $B$ in measure 2 while the formational quarter-note $E$ in measure 1 connects to the analogously formational $\mathrm{D}$ in measure 2 (see the broken horizontal lines). As shown in Figure 15, note that the cumulative eighth-notes $C$ and $B$ on level 0 in measures 1 and 2 , the points of exact return $\left(a^{0}\right)$, create a transformation that "disappears" from the analysis on level 1 since, when the analytical rules are applied recursively, these pitches on level 2 form part of a nonclosural countercumulative (c/c) durational pattern (albeit a relatively weak one: $3: 2$ ), which in turn durationally strengthens the formational $E$ and $D$ quarter notes of measures 1 and 2.

On level $2 b$, the $C \#-B$ of measures $1-2$ thus goes on to the $A$ of measure 3 , creating a descending line, whereas the $\mathrm{D}$ of measure 2 spans similarly to the $\mathrm{C}$ of measure 4. Near-return helps duration, so to speak, transform both the mildly dissonant $\mathrm{D}$ in measure 2 and the mildly dissonant $\mathrm{A}$ in measure 3 to a higher structural level. Looking ahead on level 3, we see also

47. For this reason and a lack of space, I will omit including in the analysis intervallic relationships, concentrating only on those pitch connections relevant to the discussion at hand. 


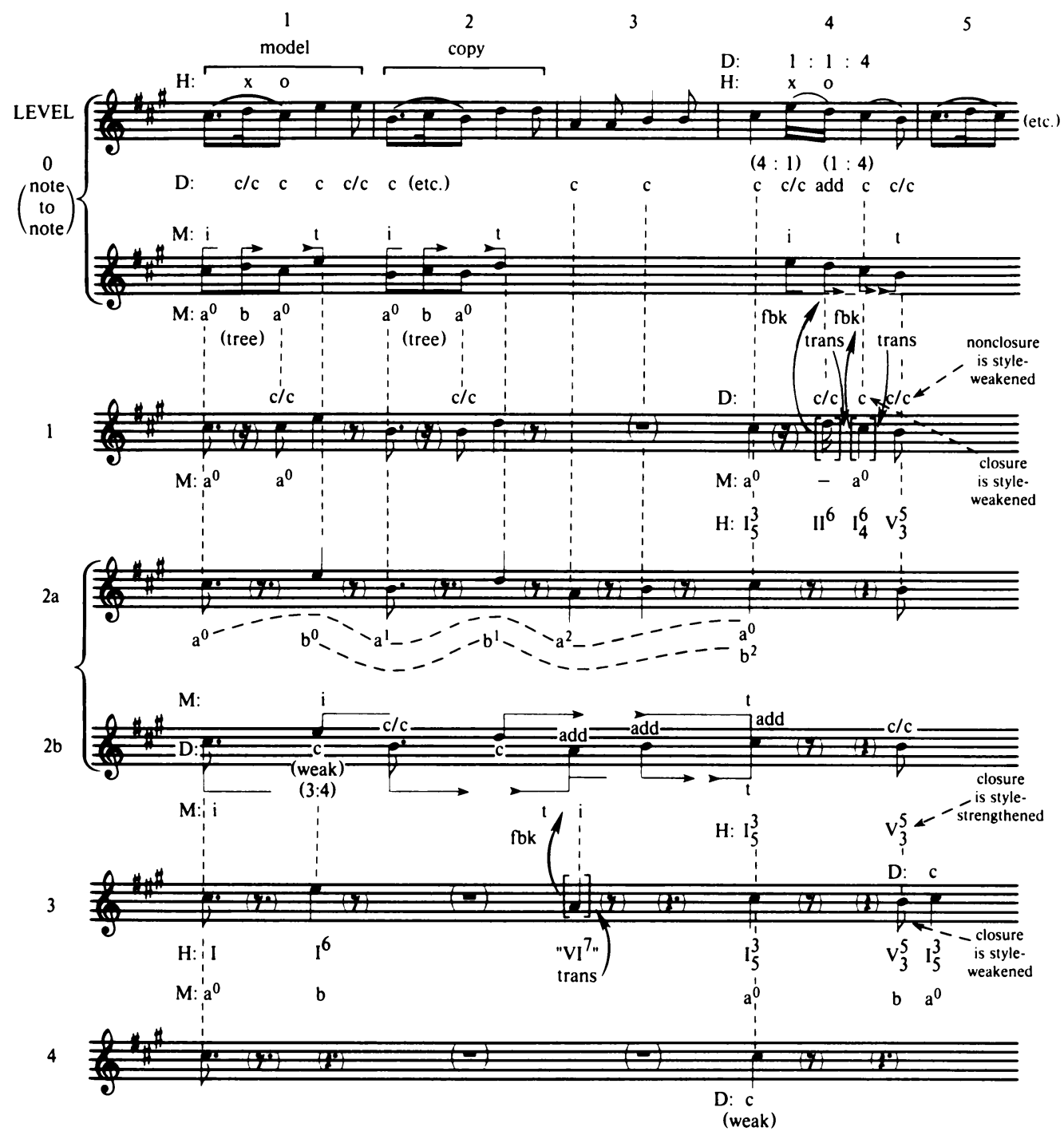

5
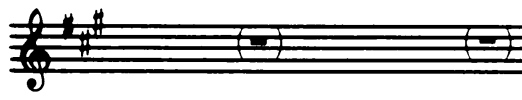

KEY

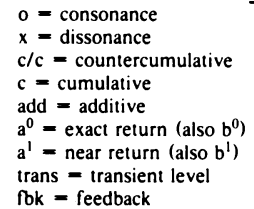

$M=\operatorname{melod} y$

$\mathrm{H}=$ harmony

$\mathrm{D}=$ duration

$\delta=$ initial (i)

$a^{0}=$ exact return (also $b^{0}$ )

trans = transient level

fok $=$ feedback

$\zeta=$ medial

$\partial=$ terminal $(t)$

Figure 15. 
that the first $\mathrm{C}$ in measure 4 connects melodically to the second $\mathrm{C}$ of the bar as an exact $\left(\mathrm{a}^{0}\right)$ return (for analytical reasons which will shortly become apparent if not intuitively so already). Note on level $2 \mathrm{~b}$ that near-return $\left(\mathrm{a}^{0}\right.$ $\left.\mathrm{b}-\mathrm{a}^{1}\right)$ creates two different descending lines ( $\mathrm{C} \#-\mathrm{B}-\mathrm{A}$ and $\left.\mathrm{E}-\mathrm{D}-\mathrm{C} \#\right)$.

We see then that registral return plays a substantial part in the formation of structural tones. Nevertheless, neither exact- nor near-return can be regarded as strong a closural force as duration or harmony. For the discontiguous registral connection to occur, other parametric closure must take place first. Thus, the cumulative (c) quarter-note $E$ in measure 1 creates the formation of a tone whose potential for further closure via the device of near-return is realized only with the cumulative quarter-note $\mathrm{D}$ in measure 2. Similarly no exact-return would occur between the two C\#'s of measure 1-no sense of a "neighboring-tone" relationship-unless harmonic and durational function closed the second $C \#$. By the same reasoning, the dissonant, countercumulative $(\mathrm{c} / \mathrm{c})$ sixteenth-note $\mathrm{D}$ of measure 1 cannot possibly connect to the similarly conforming sixteenth-note $\mathrm{C}$ of measure 2 because the nonclosural circumstances that deform both of those tones prevent them from developing any discontiguous connective potential.

\section{Parametric Applications: Initiation and Termination of Melodic Continuation}

As to the formational effect of the initiation (i) and termination ( $t$ ) of melodic patterns of continuation (as hypothesized and defined earlier), there are two discontiguous realizations of implication that occur on the lowest note-to-note level (level 0 in Figure 15): the ascending $C \sharp-D$ in measure 1 goes on to the $\mathrm{E}$ after the interruption of the reversing quarternote $\mathrm{CH}$; and the ascending $\mathrm{B}-\mathrm{C} \#$ in measure 2 stretches to the quarter-note $\mathrm{D}$ (see the analysis, where initial $=\delta$, medial $=\delta$ and terminal $=?$ ). ${ }^{48}$ Thus, the first two measures of this melody exemplify the point made earlier about music possessing both tree and network connections: the ascending linear patterns $(\mathrm{C} \#-\mathrm{D}-\mathrm{E}$; B-C\#-D) coexist horizontally as networks simultaneously with the vertical tree-patterns of exact registral return (C\#-D-C\#; B-C\#-B).

Aside from these two ascending lines, there is also a contiguous pattern of continuation on the lowest level in this example that requires discussion-the E-D-C\#-B pattern in measure 4 (again see level 0). From an analytical point of view, the configuration in this bar is very interesting. The initiating (i) sixteenth-note E functions as something of a "dramatic" event

48. An extensive discussion of the analytical symbols used in what I have called the implication-realization model can be found in my article (Narmour, in press). 
because, although it commences a pattern of continuation and is thus melodically a tone of formation, that structural potential is severely deformed by both the nonclosural countercumulative $(\mathrm{c} / \mathrm{c})$ durational pattern $(\downarrow d, \mathrm{a}$ relatively "strong" ratio of $4: 1$ ) and the harmonic dissonance $(x)$. This nonclosural combination therefore attenuates E's function as a point of initiation, decisively preventing its rise to any higher level.

But the medial D resolving the dissonance (o), and thus creating a measure of harmonic closure, does not transform either because, if the analytical rules are applied recursively, the D immediately becomes part of a strong nonclosural countercumulative $(\mathrm{c} / \mathrm{c})$ pattern on the next level $(d \AA ; 4: 1)$. Moreover, it also functions on the next level as the medial part of a harmonically nonclosural pattern-a first-inversion chord $\left(\mathrm{II}^{6}\right)$ following a rootposition chord (I) on the downbeat $\mathrm{C}$-and is thus "transient" (trans) with respect to level 1 (see the example).

At this point, we also see why both C\#'s in measure 4 connect in a pattern of exact-return (level 1). Note, however, that in hierarchical terms the C D-C\# pattern emerging on level 1 cannot be construed as a neighboring note (i.e., as $\mathrm{a}^{0}-\mathrm{b}-\mathrm{a}^{0}$ ) despite its appearing visually in connection with the transient function of the $D$ in the analysis of Figure 15 to look like one. It cannot be so because the form on the note-to-note level prevents that from happening. That is, on the surface level (0), the second $C \sharp$ in measure 4 is part of a descending E-D-C $-\mathrm{B}$ process, a form which admits of no reversal of direction to create a neighboring tone-hence, the analytical symbols of " $a{ }^{0}-a^{0}$ " instead of " $a 0-b-a{ }^{0}$." ${ }^{49}$ I shall have more to say about transience of levels shortly.

The medial $C \#$ of the descending E-D-C\#-B line in measure 4 allows us to consider the question of weighting parameters raised earlier in our discussion of noncongruence between duration and harmony. Is the relatively strong durational-closural cumulation of $1: 4$ on the second $\mathrm{C}$ in measure 4 decisive enough to offset (1) the melodic fact that this $C \#$ is a medial note (and thus nonclosural) as well as a note of exact return $\left(\mathrm{a}^{0}\right)$ and (2) the harmonic fact that this $C \#$ appears nonclosurally as part of a second-inversion chord $\left(\mathrm{I}_{4}^{6}\right)$ following a first-inversion one $\left(\mathrm{II}^{6}\right)$ (level 1$)$ as well as a second-inversion chord following a root-position one (applying the harmonic rules recursively and omitting the transient $\left.\mathrm{II}^{6}\right)$ ? Whatever decision is made about this tone should, of course, be consistent vis-à-vis the hypotheses developed earlier (p. 162) with reference to such noncongruence.

49. The "digression" in a registral pattern of return can be composed of several intervening events-in the case of the $C \sharp-E-D-C \#-B$ pitches of measure 4 in Figure 15, the $E$ and the $\mathrm{D}$ sixteenth notes function as intermediate events between the first quarter-note $\mathrm{CH}$ and its return. 
On the basis of a comparison among the relevant parameters, the decision to transform or not to transform this C would appear at best to be a standoff, at worst, inconclusive. Harmony, for example, would seem here to be moderately more nonclosural than other analogous progressions in the phrase since on level $1 \mathrm{a}$ first-inversion chord of one fundamental moves to a second-inversion chord of another fundamental $\left(\mathrm{II}^{6}-\mathrm{I}_{4}^{6}\right)$. Durational closure $(1: 4)$ on the other hand is stronger on the foreground than anywhere else in the phrase, and, as we shall see, this cumulation is further strengthened by durational feedback (fbk) from the next level-though,' to be sure, the melodic function of the $\mathrm{C}$ as a nonclosural medial tone in the linear process of E-D-C\#-B somewhat counters both these effects of duration. Exact-return on the second $C \sharp\left(a^{0}-a^{0}\right)$, as a weak closural force clashing against this, is of little decisive significance one way or the other.

However, we need not hunt up dozens of other test cases in the hopes of refining our transformational hypotheses before deciding about the levelstatus of this noncongruent $\mathrm{C}$ over the $\mathrm{I}_{4}^{6}$. Instead, we can invoke once again the factor of conformance as it appears exogenously in the style. For the interrelationship among harmony, melody, and duration in measure 4 is so commonplace in Mozart's music that the quarter-note C over the secondinversion chord cannot possibly be structural since in countless other highly conformant cases such pitches practically always resolve downward one step (to the second degree). That is, the formal "mimicry" between measure 4 and other highly conformant passages in the classical style is such that the function of nonclosure must be elevated over closure on the $\mathrm{C} \#$ despite the strong durational cumulation. Put still another way, to the competent listener the match between measure 4 and other cadential gestures in the style is so great that, in spite of the specific contextual interaction among the intraopus parameters at the cadence, the extraopus expectations generated-which is to say, the activation of the listener's prior learning-reinforces the overall function of nonclosure on the $C \#$.

The crucial criterion in invoking such stylistic arguments is that a high degree of conformance must exist between the compared examples-as in all empirical appeals which invoke replication as epistemological evidence. ${ }^{50} \mathrm{~A}$ typical highly conformant case supporting the overall nonclosural function of the $C$ in measure 4 , for example, would be the nearly identical half-cadence in measures 3-4 in the first movement of Mozart's

50. The locus classicus discussing replication and the empirical approach is Ayer's book (1946). 
Piano Sonata in $D$ (K. 576). ${ }^{51}$ Thus, though displaying a relatively high degree of durational closure, in the final analysis the $\mathrm{C}$ over the secondinversion chord in measure 4 functions only formationally (i.e., as a surfacelevel articulation) instead of as a permanent transformation since to the listener durational closure would be deformed by the high nonclosural conformance evidenced in the relevant exogenous style. As shall be discussed shortly, this kind of transformation-deformation causes transient levels.

As to the final note of measure 4 (the eighth-note $B$ ), little needs to be said. The melodic closure produced by the termination ( $t$ ) of the E-D-C\#-B pattern of continuation and the moderate harmonic closure (a secondinversion chord of one fundamental to a root-position chord of another fundamental) lie noncongruent against the relatively strong nonclosure of the countercumulative durational pattern $(2: 1)$ —resulting in a so-called "feminine" half-cadence. ${ }^{52}$ The parametric noncongruence on the B would perhaps seem to lead analytically to a kind of functional ambivalence, but in fact there is again no problem in inconsistency here with respect to our earlier transformational decisions in electing the melodic and harmonic parameters of closure to dominate over the durational parameter of nonclosure. Even if we allow the relatively strong nonclosural function $(2: 1)$ of the durational pattern to "neutralize" the closural function of the melody $(t)$, leaving only moderate harmonic closure, the invocation of extraopus style again ensures that closure will be elevated over nonclosure with respect to the $B$ since the use of a dominant chord to end an antecedent phrase is exceedingly common in this style. ${ }^{53}$

\section{Hierarchical Feedback, Vertical Networks, and Transient Levels}

I have presented a relatively exhaustive analysis of measure 4 not only to demonstrate how a parameter-by-parameter analysis might work in the

51. This example is conformant in practically every way except tempo:

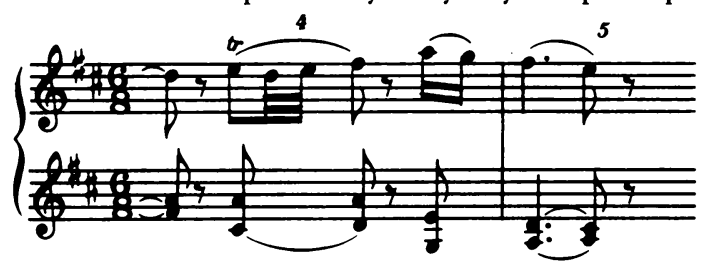

52. Melodic reversal takes place because the consequent phrase (measures 5-8) starts on the same $C$ which began the antecedent phrase (measures 1-4).

53. Note that the effect of style varies from one level to another. On level $2 b$, style strengthens the closural aspect since ending on $\mathrm{V}$ in the antecedent phrase, as mentioned, is all but a cliché. On level 3, however, style weakens closure since from the aspect of the consequent phrase (beginning on measure 5) the function of the $\mathrm{V}$ to imply and go on to connect to the $\mathrm{I}$ is also a cliché of the style. 
recursive generation of transformational levels but also to concretize further how a hierarchical approach differs from a systemic one. As we have seen, a true hierarchical approach insists on partially decomposable levels-where analytical functions paradoxically are dependent on, yet independent from, the transformations that actually take place. With respect to an artifactual phenomenon like music I have hypothesized further that dualistic properties like this exist simultaneously, in the form of parametric closure versus parametric nonclosure. If this is correct, then such noncongruence increases the probability that the syntactical relationships in a piece of music will occur discontiguously, creating hierarchical networks in addition to hierarchical trees since what happens nonadjacently in retrospect may alter what happened in prospect. We saw, for example, such discontiguous relationships in the downbeat motives of measures 1 and 2 in the Mozart phrase and how they added horizontal networks to the tree structures.

Network effects in musical hierarchies, however, do not only occur "horizontally" across time. They also take place "vertically" from high level to low level through the operation of "feedback." Indeed, because in hierarchies levels are only and always partially decomposable, the normal separation existing between a high level and its adjacent low level may be so attenuated by such feedback that what appears transformational in prospect from analytical evidence on the low level may become deformational in retrospect because of what occurs on the higher level. When this happens, when a transformation exists only temporarily-when a closural function on a low level leaps to a new level only to collide with a nonclosural function there and thus ricochet back to its original level-we may quite properly speak of transient levels.

Transience is the result of a "vertical" perceptual revision necessitated by deformation that takes place on the level of the transform. Sometimes this retrospective deformation is so strong that the emerging level becomes immediately downgraded. Thus, unlike systemic levels where reduction is always from low level to high level, in hierarchies we must also admit the opposite possibility of reduction from high level to low level. ${ }^{54}$

To see how this works, let us again turn our attention to measure 4 of the Mozart phrase (Figure 15). Now despite all that has been claimed in this paper about the importance of partial decomposability with respect to "true" hierarchical levels and the theoretical necessity of conceptualizing parametric closure and nonclosure as potentially simultaneous analytical possibilities, the reader might wonder how essential these assumptions are

54. Thus, the terms "prospect" and "retrospect" must be applicable in music "vertically" with respect to levels as well as "horizontally" with respect to time. 
since, if the brackets in Figure 15 are ignored, the level-analysis of measure 4 looks rather systemic, as the following tree display of the melody indicates:
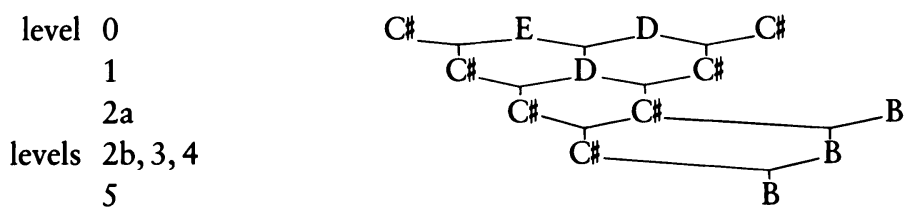

But though, as I mentioned, the whole Mozart phrase does seem about as tree-structured as an analytical example can get, such a simplistic, systemic display of the melody in measure 4 analytically ignores the partialness of levels and therefore is oblivious to what really happens hierarchically.

For in a true hierarchy, just as congruence in closural function from transformation to transformation increases the strength of a given level and thus "feeds forward" up the hierarchy to ever-progressing stability, so congruence in nonclosural function from transformation to transformation decreases the strength of a given level and thus feeds back down the hierarchy, reinforcing instability. Thus in measure 4 of the Mozart example, the sixteenth-note $\mathrm{D}$ on the surface level is in prospect part of an additive durational motion $(1: 1)$ whose weak nonclosure in this respect is largely offset by the relatively strong harmonic closure on the surface level as the dissonant $\mathrm{E}(\mathrm{x})$ resolves in prospect to the $\mathrm{D}(\mathrm{o})$. It is this harmonic closure that causes the transformation to level 1 in the first place.

However, since in real hierarchies events are not absorbed by the levels they create but rather always carry their total identities with them, the D must retain its durational, harmonic, and pitch properties as well as its syntactic functions as it moves to a higher level. Thus, on the next level (1) the $\mathrm{D}$ is again countercumulative $(\mathrm{c} / \mathrm{c})$ in conjunction with the quarter-note $C \#$ at the beginning of the bar. And this nonclosural countercumulation, moreover, is prospectively operational with respect to the surface level. ${ }^{55}$ That is, the countercumulation which emerges on level 1 immediately feeds back to the lowest level, altering in prospect the durational meaning of the $\mathrm{D}$, which appears in the actual music to be additive and thus only a weak case of durational nonclosure.

Hence, though on the note-to-note level the $\mathrm{D}$ in connection with the

55. One of the most difficult problems in music analysis is dealing with the effects of higher levels on lower levels. In the case of D's prospective meaning in following the dissonant $\mathrm{E}$, it is heard additively and consonantly as a resolution. The resolution brings about the transformation to level 1 . Once achieved, the tranformation forms a relationship with the $\mathrm{C}$ at the beginning of measure 4 , the last tone transformed prior to the $\mathrm{D}$. Now the $\mathrm{D}$ in relation to the preceding $C$ suddenly becomes countercumulative. 
preceding $\mathrm{E}$ looks to be prospectively additive in terms of duration (1:1), this is not the only way it functions because the congruent, nonclosural, hierarchical, durational function of level 1 (c/c) feeds back (fbk) "vertically" into the D on the low level. Thus, from the aspect of the parameter of duration, the $\mathrm{D}$ functions almost simultaneously (once the transform emerges) as both additive (1:1) and strongly countercumulative $(4: 1)$. Moreover, the positive generative effect of high-level nonclosure feeding back congruently to low-level nonclosure so deforms the $\mathrm{D}$ as a melodic note that its appearance on level 1 becomes transient (symbolized by "trans" and the brackets). In other words, vertically the D's transformative function in prospect becomes downgraded in retrospect, creating a complex network between the two levels. ${ }^{56}$

A major difficulty in developing a hierarchy theory for music analysis is thus not only to discover rules of level-transformation but also to discover the rules governing level-transience. What degree of congruent nonclosure between levels is sufficient to downgrade a hierarchical transformation? Put conversely, when closure and nonclosure "conflict"on both the low level and the transformed level, how strong must the closure be to ensure the permanence of the transformation? How can we keep simultaneous track of both vertical and horizontal influences on level-relationships? The question of time-dependent feedback cannot be avoided since a cardinal tenet of hierarchical theory is a nonidealized concept of time. Time enters into vertical prospective and retrospective relationships of closure and nonclosure as surely as it does into horizontal syntactic relationships. Hierarchical networks operate "up and down" as well as "across." Were this not so, we could be content to see the pitches of the E-D-C measure 4 as either the simplistic tree of page 174, which is absurd in the face of the perceptual unity of the gesture, or as one unreduced linear unit like that of level 0 , which is equally absurd in view of the various parametric interactions of closure and nonclosure.

With what has been presented so far, the reader may go on to examine in Figure 15 the analysis of the higher-level functions of the phrase, seeing how a recursive application of the analytical rules for the parameters of melody, harmony, and duration would bring about the emergence of hierarchical levels. Observe, as emphasized, that notes retain both their registral and

56. Note, however, that in a separate-parameter approach the melodic $\mathrm{D}$ can be transient without the $\mathrm{II}^{6}$ harmony functioning in the same way. For there is nothing that happens on level 2 to deform the harmony. There is thus counterpoint between the melody and the harmony on the $\mathrm{D}$ in that the level-strength of each parameter is noncongruent. As we shall see, a typical systemic analysis would evaluate the $\mathrm{II}^{6}$ of measure 4 and its counterpart in measure 8 in the same reductive light. But hierarchically we see that the transient D in measure 4 is significantly different melodically and harmonically from the nontransient $D$ in measure 8 . 
durational not to mention harmonic identities on higher levels; a dotted eighth note which appears on the whole-note level is still a dotted eighth note. Note especially on level $2 b$ the high-level patterns of continuation, discussed earlier. Observe also on level 2 how the medial functions $(\rightarrow)$ of the $B$ on the downbeat of measure 2 and the $B$ on the second beat of measure 3 prevent both these tones from establishing any higher-level registral patterns of return. And note as well on level 3 the effects of both higher-level harmonic process and durational cumulation and countercumulation on the pitches transformed. Finally, note that because the harmonic nonclosure of the A over the "VI"' in measure 3 increases on level 3, following after the $\mathrm{E}$ over the $\mathrm{I}^{6}$ in measure 1 on that level, hierarchical feedback (fbk) again occurs. Thus the A's appearance on level 3 is only temporary (trans).

It may be observed again that the higher we progress analytically through the levels of Figure 15, the more rationalistic and compositionally structural the levels seem to become. The last three levels, for instance, seem quite divorced from our perceptual experience. The operation of recursion thus has its limitations as regards the cognition and perception of music.

\section{The Problems with Reductive-Systemic Alternatives}

For purposes of comparison, it will now be instructive to examine very briefly four other analyses of Mozart's theme-by DeVoto, Lester, Schenker, and Meyer. ${ }^{57}$ The point here is not just to criticize each analysisthough that cannot be avoided-but rather to throw into relief the empirical-analytical difficulties that come about when compositional structures and systemic trees are elevated over hierarchical networks and partially decomposable levels. As shall be seen through the various reductive-analytical symbologies, each of these rather different analyses encounters problems because each in one way or another invokes a generational system of analytical rules biased toward fully decomposed levels. That the reductivesystemic approach applied to a theme as simple as Mozart's produces such significantly different analyses-each with different analytical problemsargues that it is time to rethink our concepts as to what musical levels are, how they come about, and what their true hierarchical organization is.

Figure 16 shows a voice-leading analysis of the whole theme by DeVoto whose method is to "reduce" the melody by eliminating the "ornamental details" on each successive level. ${ }^{58}$ I have chosen to introduce the systemic

57. There are numerous other analyses of the Mozart example that the reader may wish to examine. A few that come to mind are found in Cone (1968, pp. 28-31), Morgan (1978, pp. 440-451), and Lerdahl and Jackendoff (1977, pp. 111-171).

58. The example is found in Piston (1978, p. 102), revised and expanded by DeVoto. 
approach with this analysis because it typifies the kinds of difficulties one encounters in trying to generate structural levels-how to weigh parameters, how to balance form against function, how to disentangle specific cases from the general style, how to coordinate trees with networks, how to apply analytical rules consistently, and so forth.

We will concern ourselves primarily with the first four bars. The reduction of the original theme to level $a$ is accomplished by removing the repeated notes (measures 1, 2, 3, etc.). ${ }^{59}$ In view of the arguments presented in connection with Figure 15, this seems reasonable since these counter-cumulative tones are durationally "open" and unclosed. To move from level $a$ to level $b$ requires two operations-tagging the neighboring tones ("N," measures $1,2,4$, etc.) and the dissonances (the appoggiatura in measure 4 )-and then omitting them from level $b$. Transformationally, the analysis implies that the $\mathrm{C}-\mathrm{E}-\mathrm{D}-\mathrm{C}-\mathrm{B}$ in measure 4 on levels $a$ and $b$ is a completely decomposable tree-structure, not unlike the one discussed earlier: ${ }^{60}$

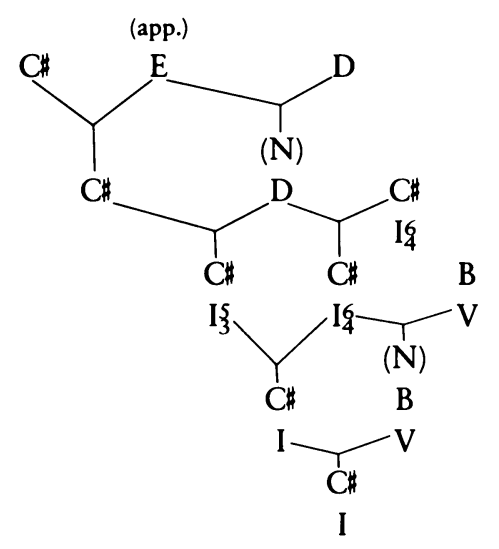

59. Some theorists would say the repeated tones are to be omitted because they are "echoes" or unimportant afterbeats (nonaccents).

60. I say "implies" because DeVoto's analysis is not this systematic. A recursive evaluation leads to the following inconsistency with respect to the N's:

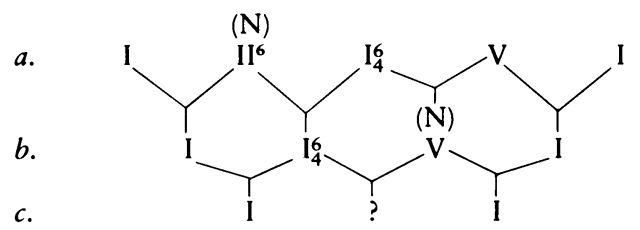

If the $\mathrm{II}^{6}$ is reduced out on level $b$, then so must the $\mathrm{V}$ on level $c$. Put another way, if the $\mathrm{I}_{4}^{6}$ is more structural than the $\mathrm{II}^{6}$ because the $\mathrm{II}^{6}$ is an "N" or because I is a tonic, then the $\mathrm{I}_{4}$ also must be more structural than the $\mathrm{V}$ which is also an "N" and a nontonic. DeVoto's analysis wants it both ways, so levels $b$ and $c$ fudge the issue. 

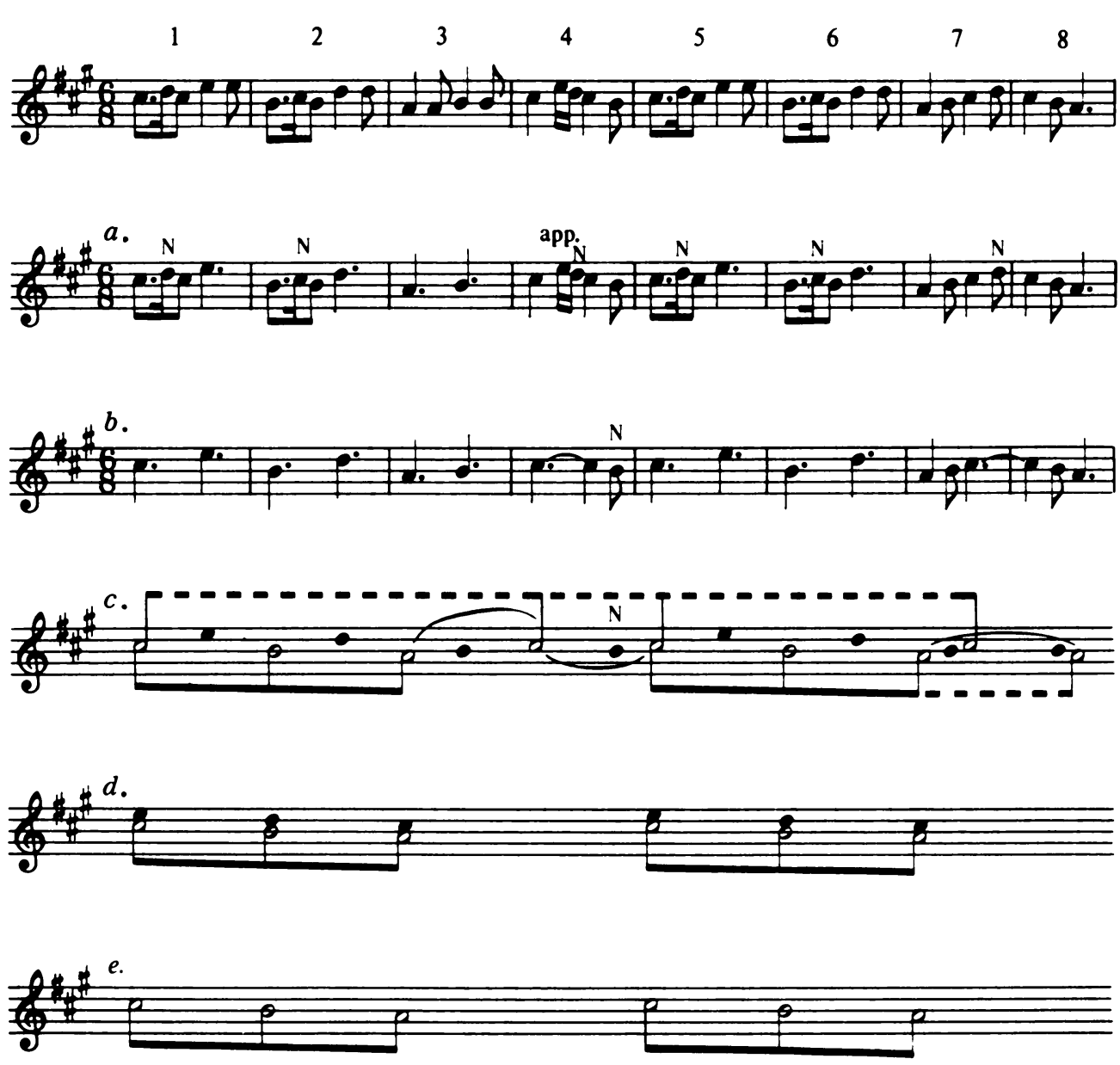

Figure 16. After DeVoto.

The analysis, however, comes quickly to grief because DeVoto concludes on the highest levels $(d$ and $e$ ) that the first phrase (measures 1-4) and the second phrase (measures 5-8) are alike in melodic structure (both C\#-BA). This is obviously rather indefensible since in measure 4 both the $C \sharp$ (over the I) and the B (over the $\mathrm{V}$ ) are harmonically more stable as root-position triads than the A in measure 3, a "VI" occurring over a dominant pedal.61 The faulty analysis comes about because DeVoto sees the C over the tonic

61. DeVoto's analysis in this respect has also been criticized by Hasty (1982, pp. 158160 ) in a review of Piston's Harmony. 
on the downbeat of measure 4 as an "arpeggiation" of the A in measure 3, which relegates the C to a stemless black note on level $d$. Hence the untenableness: the chord on the downbeat of measure 3 erroneously comes to be more structural than the tonic on the downbeat of measure 4. Moreover, in terms of the tonal closure of the style, it seems quite mistaken to assert that the two phrases are exactly alike melodically since the first phrase ends on a dominant half cadence and the second on a tonic full cadence.

These problems arise partly from generating in measure 4 a completely decomposable tree-structure on level $a$ instead of a partially decomposable network-hierarchy where, for instance, the downbeat of measure 3 can function as a closural transform in prospect but a transient temporary event in retrospect. Further, it is more probable that the E-D-C $-\mathrm{B}$ melodic line in measure 4 , as I discussed, is perceived as one partially decomposable process with melodic and harmonic closural articulations on the $\mathrm{E}$ as an initial tone, on the $\mathrm{D}$ as a consonant resolution, on the $\mathrm{C}$ as a durationally cumulative tone, and on the B as a resolution of the ${ }_{4}^{6}$ chord (as shown in Figure 15) -rather than as a simplistic tree. ${ }^{62}$

Aside from these problematic level-transformations, there are also inconsistencies in the application of the analytical rules in Figure 16 which result from trying to force a hierarchical relationship into a systemic-tree mold. For example, in order to continue generating levels from $b$ to $c$ DeVoto constructs on level $c$ a "notational hierarchy" of white and black notes which takes into account "metric values." 63 Thus, the black notes become either "arpeggiations" to the white notes; or passing tones (the B's beneath the slurs, measures 3-4 and 7-8); or neighboring tones (the B in measure 4, already discussed) ${ }^{64}$ In addition, DeVoto connects all the downbeat white notes with beams and all the repeated C\#'s (and the two A's at the very end) with "dashed beams." With these new rules, as we saw, the analysis can now be moved to levels $d$ and $e$ since the black notes (passing tones and neighboring tones) can be omitted, the arpeggiations can be reduced to chord tones over the white notes, and the repeated white notes (i.e., the C\#'s) can be taken out. Note incidentally that in this systemic analysis the transformed events lose their notational identity along with their inherent low-level functions as they are decomposed and reduced to higher levels.

Since real hierarchies are discontinuous, one should, of course, admit the

62. The $\mathrm{E}$ in measure 4 is not as dissonant as ewould ordinarily be the case were the fifth of the implied chord $(F \#)$ present because then the $E$ would grind not only against the $D$ in the bass, creating a second, but also against the $F \#$, creating a seventh. The full harmony may be seen back in Figure 11.

63. DeVoto (1978, p. 102).

64. DeVoto (1978, pp. 102-103). 
possibility that different criteria may be necessary for generating succeeding levels. DeVoto's rules for levels $c, d$, and $e$, for example, are in large measure different from those of $a$ and $b$. But the analysis becomes problematic because the transformational rules invoked for levels $c$ and $d$ are contradictory to those utilized for generating levels $a$ and $b$. For instance, if the B of measure 3 on level $c$ is a passing tone, then why isn't the B of measure 2 one also-if not on level $d$, then at least on level $e$ ? That is, if the transformational principle of "passing tone" to get from level $c$ to $d$ is applicable to the $\mathrm{A}-\mathrm{B}-\mathrm{C} \#$ line of measures $3-4$, then why not also apply it to the $\mathrm{C} \#-\mathrm{B}-\mathrm{A}$ of measures 1-2 to get from level $d$ to $e$ ?

To take another example of inconsistency, if "metric value" is a criterion for generating the white-note structural tones on level $c$, then why is the $\mathrm{C} \#$ of measure 7 on the weaker beat 2 the transformed tone, instead of the $\mathrm{C \#}$ in measure 8 on the stronger beat 1 ? That the beginning of measure 8 (see Figure 11) is over an unstable $\mathrm{I}_{4}^{6}$ (in contrast to the root-position I on beat 2 of measure 7) does not resolve the inconsistency since harmonic instability over the downbeat $\mathrm{A}$ in measure 3 did not deter DeVoto from transforming the latter tone. Obviously, if one opts for generating only tree-structured levels in musical analysis, then recursively applying the analytical rules in a consistent way becomes obligatory unless other formulated constraints against such recursion are spelled out.

There are other voice-leading approaches which essentially generate tree structures but which are more internally consistent in their higher-level reductions-and thus whose transformations are more convincing-than the ones presented by DeVoto in this analysis. Schenker's analysis of the same piece, for example, is more plausible because the controlling reductive principles of the analysis are tranformationally conceived such that the possibility of contradictory rules arising is considerably lessened. As we shall see, however, the advantage gained in this theory of transformations comes at a considerable cost since the higher-level rationale of the theory tends to distort the networked, partially decomposed level-relationships found in musical hierarchies.

Figure 17 shows the analysis of the phrase under discussion. ${ }^{65}$ Compositional structures in the shape of voice-leading transforms are used as a means to generate the structural levels whereby foreground complexities are progressively reduced into increasingly simpler relationships. Although much has been made of the hierarchical nature of Schenkerian analysis, as

65. The example is compiled from three separate analyses in the Anhang of Schenker's Der freie Satz (1956): level 1 comes from example 141-d (p. 101); level 2 from example 875 (p. 43); level 3 from example 132-6 (p. 93). I have omitted certain insignificant details and added the numbers in the brackets on level 3 . 

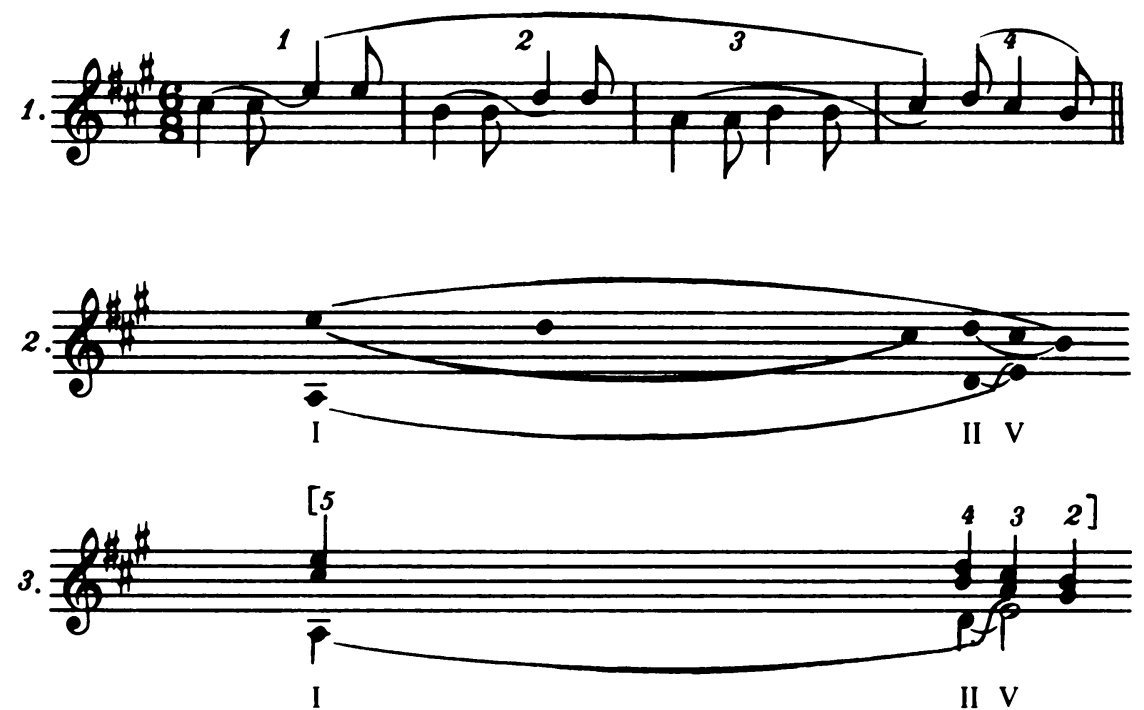

Figure 17. After Schenker.

we shall see from the analytical problems discussed, in reality it exemplifies a systemic approach.

Like DeVoto's analysis, Schenker's begins on the lowest level (1) without the neighboring tones $\mathrm{D}$ and $\mathrm{C}$ of measures 1 and 2 and the appoggiatura $\mathrm{E}$ in measure 4. But unlike DeVoto, Schenker's systemic levels are not derived from the bottom up but from the top down. That is, Schenkerian analysis is "concept-driven" instead of "data-driven." And like all such systems, an immutable initiating higher-level principle must always exist prior to the levels generated. Thus, for instance, instead of asserting metric downbeats as a level-differentiating criterion, Schenker elects to organize levels around descending lines and "fundamental progressions," specifically in measures 1-4 of Figure 17 a 5-4-3-2-Urlinie over the I-II-V chords on the highest level, beginning on the $\mathrm{E}$ on the weak beat in measure 1 and going to the "interrupted" eighth-note B on the half cadence in measure 4 (see level 3 of Figure 17). ${ }^{66}$ Once the Urlinie is determined, Schenker can then go on to assert that the $\mathrm{E}$ (the 5) governs the melodic pitches from measure 1 to the beginning of measure 4 , being prolonged by the lower-level descending line of E-D-C\# (level 2 in the example). Hence, in his terms, the pitches on level 2 from measure 1 to the beginning of measure 4 become either "inside" voices or "outside" voices of the asserted descending line of organization, 


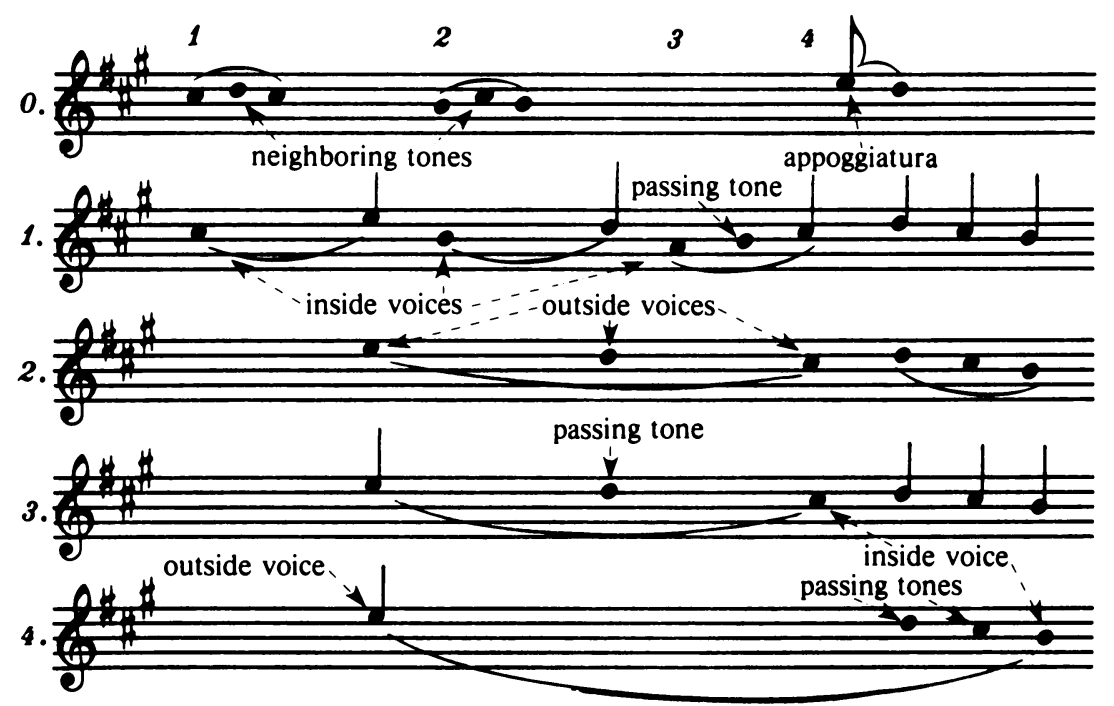

Figure 18.

the exception being the $B$ of measure 3 which would be labeled as a passing tone. The stages by which this is accomplished are shown in Figure 18.

Schenkerian theory thus derives the function of the pitches and the levels on which they appear by asserting a priori fundamental lines and harmonies and then seeing a posteriori how the various voices elaborate the foreordained theoretical premises. Of course, the type of high-level "primordial structure" elected to illuminate any given piece is not plucked out of the thin air but is chosen and "positioned" onto the music according to what seems most consistent, most relevant, and most convincing.

However much better Schenker's systemic analysis is than DeVoto's, it is not without serious problems. They result on the one hand from a theoretical insistence that only certain immutable kinds of linear-harmonic forms (Ursatz ones) be allowed to generate the structural levels and on the other hand from a decision to ignore, or at least relegate to a distinct subsidiary status, the structural role form, duration, and the other relevant parameters play in creating the level-transformations. Moreover, the employment of only certain types of harmonic voice-leading constructs to generate levels from the top down results not in a hierarchy but a systemic tree where levels are thoroughly decomposed, as Figure 18 shows.

Because of such a tree system, for instance, Schenker argues that the phrase is constructed melodically on two similar third-progressions (Züge), an E-D-C\# from measure 1 to the begining of measure 4 and a D-C $-B$ for the remainder of measure 4 (see again Figure 17) which, when combined, 
make up overall the high-level descent of the fourth-progression (e.g., E-D-C $-\mathrm{D}-\mathrm{C} \#-\mathrm{B}) .{ }^{67}$ Hierarchically, however, this analysis of a chain of two third-spans combined into one fourth-span makes little sense.

First of all, there is the problem of form. For in a partially decomposable hierarchy of levels, as will be recalled from Figure 15 and the discussion throughout, the lower level functions of transformed events do not disappear, becoming "reduced out" as one moves to higher levels. Rather, since in musical hierarchies low levels literally create higher ones, the functions of events on low levels become embodied in the events of higher levels. Were this not so, vertical and horizontal networking would not occur. Nor would feedback. This is why one says that hierarchical levels are always partially decomposable. And for a formal similarity to exist between two patterns on a higher level, there must first occur functional similarity on the lower level such that the higher-level patterns owe their emergence to similar lowerlevel formation. That any two structures-such as the ascending thirds in Schenker's analysis-look analytically alike on any given level in a true hierarchy is totally irrelevant unless the parametric formations contributing from the levels below are also conformant.

If this were not true, form would have no significant effect on the perception of music and would be of little interest either to composers or music analysts. ${ }^{68}$ Recall that the meaning of the higher-level pitch pattern in Figure $1 \mathrm{a}$, where lower-level conformance is $\mathrm{A}^{0}-\mathrm{A}^{1}-\mathrm{A}^{2}$, for instance, is very different from the meaning of that in Figure 1b, where low-level conformance is $A+B+C+D$, despite the fact that in a systemic sense similarity exists between the pitch structures of the two examples on the higher level when both are viewed "linearly," that is, when the higher levels are disembodied from their hierarchical origins on lower levels.

To return to Schenker's analysis, clearly the real low-level forms underlying the pitch structures of measure 1 to the beginning of measure 4Schenker's overall E-D-C\# progression-are so nonconformant in comparision with the mere articulations from there to the end of measure 4Schenker's D-C\#-B progression-that making a melodic relationship of two similar third-progressions is irrelevant-at best an observation of either a style form or a compositional structure. The third-progression assumption comes about, of course, because of Schenker's insistence that the fundamental line must be 5-4-3-2. And once the 5 is elected to occur on the $E$ of measure 1 , the 4 (on the D) thus has to follow in measure 4 since only there

67. Schenker (1956, p. 189).

68. Nor would variation form, which gets its aesthetic power from the hierarchical meaning of conformance and nonconformance, have ever emerged as one of music's heartiest compositional strategies, regardless of culture. 
does the harmony (on the $\mathrm{II}^{6}$ ) appear in accord with the a priori Ursatz stipulations of the theory.

In terms of symbology there is moreover the problem of treating the $\mathrm{E}-$ $\mathrm{D}-\mathrm{C} \#-\mathrm{B}$ line in the music of measure 4 as simply $\mathrm{D}-\mathrm{C} \#-\mathrm{B}$ on a higher level. For how can $\mathrm{D}$ on the note-to-note level be denied its function as a medial tone in the E-D-C $\#-B$ melodic process and its medial function as a sixteenth note in both an additive and countercumulative durational pattern (which cumulates on the ensuing quarter-note $\mathrm{C}$; r recall Figure 15)? Even harmonically the D never functions as an initial tone, only as a terminal tone following the dissonance on the lowest level or as a medial tone in the $\mathrm{I}_{-1{ }^{6}}$ $\mathrm{I}_{4}^{6}-\mathrm{V}$ progression on the next level. The point is, $\mathrm{D}$ simply does not function as an initial tone in any parameter. Even laying aside the theoretical concepts of initial, medial, and terminal, melody and duration create the level-identity of the $\mathrm{D}$ as much as the fact that it is the resolution of a dissonance. In a true hierarchy, of course, all these attributes would remain with that note as it traveled through higher levels. One feels in Schenkerian analysis that if melody and duration were taken into account as separate parameters and if musical levels were conceived as hierarchies instead of as systems, one would not assent to the Ursatz premise in general-not to mention the specific 5-4-3-2 line in this analysis-quite so readily. ${ }^{69}$

There are other problems with Schenker's analysis which seem to derive mostly from the high-level generation of a tree-structure system via the a priori rationalistic premises upon which the theory is based. For example, most listeners hear the tonic chord in the position of the third at the beginning of measure 4 as being a tonal, melodic, and harmonic return to the chord which began the piece. But because Schenker's levels are completely decomposed, his tree analysis has no symbological provision for connecting these pitches. The $\mathrm{C}$ in the melody in measure 1, for instance, cannot be joined in the analysis to the $C \#$ in measure 4 except as an inside voice below the foreordained 5 (the $\mathrm{E}$ in measure 1 ). The systemic nature of the theory simply does not allow for such discontiguous relationships.

For that matter, Schenker's tree analysis perforce severs certain kinds of manifestly present contiguous relationships, treating them as if they were not there. Apart from being voices of harmonic motion, for instance, what explains the purely melodic relationships between the $\mathrm{E}$ of measure 1 and the contiguous $\mathrm{B}$ of measure 2, or the D of measure 2 and the contiguous A of measure 3 , or the $C \#$ and the contiguous $E$ of measure 4 ? Schenkerian

69. Note that Schenker does not call the $\mathrm{D}$ in measure 4 a neighboring tone as DeVoto did since to do so would have ruined the 5-4-3-2 Ursatz. It is very peculiar, incidentally-and a tip-off to a systemic, nonhierarchical analysis-that theorists maintain the exact pitch registers of a lower level when transforming them to a higher level but rarely maintain exact durational properties. 
theory is either silent about explaining such matters, since the level-analysis ultimately decomposes all melodic relationships into harmonic voices, or else pretends that such relationships do not exist. ${ }^{70}$

Given these and other similar problems, we are thus justified in raising questions about the fundamental premises of this analysis. Other than the usual special pleading for accepting the tenets of the theory on faith, there seems little parametric justification to elect the 5-4-3-2 Ursatz as the fundamental generating principle in Mozart's phrase since the $\mathrm{E}$ in measure 1 , which is Schenker's 5 , is harmonically more open over the first-inversion chord than the preceding quarter-note $\mathrm{C} \#$ over the root-position chord in measure 1 -or the analogous chord at the begining of measure 4 , as we saw in Figure 15. Of course, one problematic or mistaken analysis does not necessarily invalidate the whole theory. Within Schenkerian theory, there are alternatives to Schenker's rationalistic and rather unconvincing analytical choices for the structural tones of Mozart's theme.

Adhering to the basic Schenkerian approach to generate structural reductions on various levels, Lester, among others, for instance, has argued for mapping a different fundamental line onto the phrase. As can be seen from Figure 19, he opts for a descent from the opening $C \#$ in measure 1 to the $B$ on the half cadence in measure 4 (level 3 ) instead of Schenker's $E$ to the B. In other words, Lester elects a 3-2 Urlinie to organize the pitches in measures 1-4 from the top level downwards. ${ }^{71} \mathrm{He}$ does so partly for the objections just listed in connection with Schenker's analysis, namely the E's (Schenker's 5) lack of "harmonic support" in measure 1-but also because the melodic $A$ and $B$ in measure 3 are harmonized in parallel tenths with the bass voices $\left(\mathrm{F} \#-\mathrm{G}\right.$, see measure 3 of Figure 19)..$^{72}$ The choice of the 3-2 line for the first four measures certainly seems more reasonable than Schenker's far-fetched descent from the E, but at the same time, because of the tenets of Schenkerian theory, it forces Lester to take an equally implausible view about the A on the downbeat of measure 3 (what in Figure 15 was tagged as a transient event on level 3).

To understand why takes a little explanation. Schenkerian theory, as is well-known, begins with the belief that artworks are completely unified

70. Such a claim, for example, is made by Salzer, a disciple of Schenker, in his book (1962, I, p. 41). For a further discussion on such severed melodic relationships, see Narmour (1977, pp. 68-73).

71. The example is from Lester's article $(1979, \mathrm{p} .75)$. Both Lester and Schenker see the whole phrase as exemplifying an interruption of the fundamental line. Schenker says the overall pattern is 5-4-3-2//5-4-3-2-1; Lester, 3-2//3-2-1. Morgan's analysis (1978) and Cone's (1968) agree with Lester's.

72. Lester (1979, p. 76). 
1

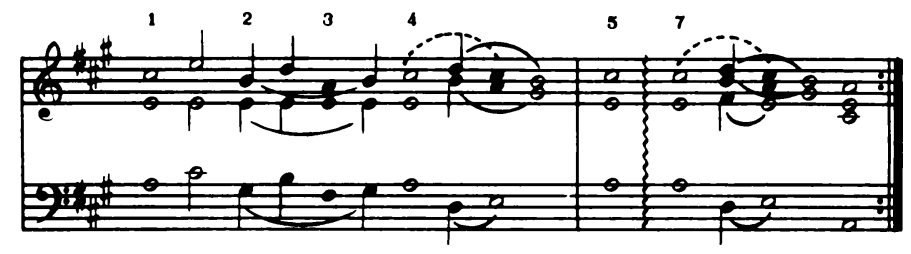

2

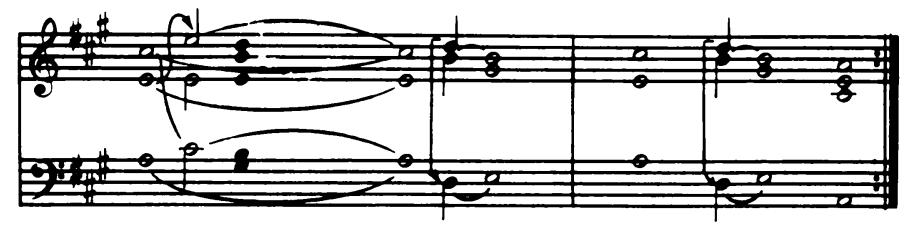

3

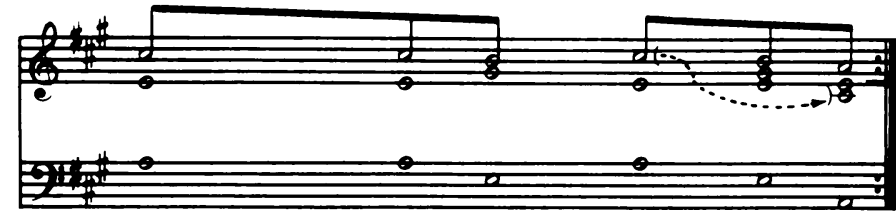

Figure 19. After Lester.

entities and that the source of this unity resides in tonality. Thus, pieces are viewed as prolongations, expansions, elaborations, etc. of the tonic chord (I), the Ursatz being the "primordial" structure spelling out the tonality. From the assertion of the fundamental progression (I-V-I) in its three descending voice-leading guises $(8-1,5-1,3-1)$ come all the rules of transformation-those governing neighboring tones, passing tones, outside voices, inside voices, and so forth-which usually result in tree-structural, systemic reductions. ${ }^{73}$

Within the theory, there is thus a strong structural bias toward the stipulated tonic (I) on the one hand and the determinative dominant $(\mathrm{V})$ on the other. Eventually in a Schenkerian analysis, all the voices of a piece of music are relegated to, and become the servants of, one or the other of these two chords. In fact, ultimately, every note is seen in light of how it prolongs the tonic. Thus, in Schenker's analysis (explicated in Figure 18), the chord on the downbeat of measure 3-the $A$ in the soprano over $F \#$ in the bass with an $\mathrm{E}$ pedal in the tenor-becomes a prolongation of the tonic, as unfolded in the structural tree (level 1). That is, this melodic A is an "inside voice" to

73. The general approach with respect to level-analysis is criticized in Narmour (1977, chapter 8 ). 
the $C \#$ over the root-position I on the downbeat of measure 4, the $C \#$ ultimately being part of the latent descending line of E-D-C\# (level 3). Because to Schenker the $\mathrm{D}$ of the latter pattern is a passing tone (level 3 ), the $\mathrm{C}$ on this level itself becomes an "inside voice" to the structural E (the 5) of measure 1-and so on, up the analytical tree to the highest level. (See again Figures 17 and 18.)

Now Lester has difficulty subscribing to Schenker's view that the downbeat of measure 3 serves to prolong the downbeat tonic of measure 4 with the $\mathrm{CH}$ in the soprano. He argues instead that the chord in question in measure 3 functions at the service of the dominant $(\mathrm{V})$ rather than the tonic (I). Thus, as can be seen in Figure 19, he analyzes the A in the soprano of measure 3 on level 1 in terms of Schenkerian theory as a lower neighboring tone lying between the $\mathrm{B}$ over the $\mathrm{V}^{6}$ at the beginning of measure 2 and the $\mathrm{B}$ over the $\mathrm{V}^{6}$ at the end of measure 3 (indicated by the slur). In turn, these two dominants can be collapsed systemically on level 2 into one first-inversion $\mathrm{V}_{5}^{6}$ chord. That is, the $\mathrm{B}$ on the downbeat of measure 2 and on the second beat of measure 3 (an "inside" voice) together with the "arpeggiated" $D$ in measure 2 in the top voice (an "outside" voice) become a passing, neighboring-tone construction between the arpeggiated $\mathrm{E}$ of the opening tonic over the $\mathrm{I}^{6}$ in measure 1 and the return of the tonic at the beginning of measure 4 (see Figure 19, level 2). The $\mathrm{I}^{6}$ in turn is an arpeggiation of the $\mathrm{I}$ on the downbeat in measure 1, serving in retrospect on level 3 to prolong the $\mathrm{CH}$ there. $^{74}$

Hence, in contrast to Schenker's tree analysis of levels, which melodically nests one inside voice (the A of measure 3 ) to another (the $\mathrm{CH}$ of measure 4) to another (the $\mathrm{E}$, the 5 back in measure 1), Lester's analysis nests a neighboring tone (the $\mathrm{A}$, measure 3 ) within a neighboring tone (the $\mathrm{B}$, measure 2 ) to the prolonged $\mathrm{C}$ spanning measure 1 to measure 4 . This is shown in the tree display of Figure 20 which should be compared with that of Figure 18.

Though the high-level construction of the melody of measures 1-4 moving basically from $\mathrm{C}$ (the 3 ) to $\mathrm{B}$ (the 2 ) might seem more convincing than Schenker's 5-4-3-2 organization, Lester's analysis presents its own set of difficulties. From a traditional Schenkerian perspective, for instance, there is the obvious inconsistency that, if the arpeggiated pitches of the dominant prolongation spanning measures 2 and $3\left(\mathrm{~V}_{5}^{6}\right)$ are to be harmonically verticalized (as shown on level 2 of Lester's analysis), so that the $E$ in measure 1 is connected to the $\mathrm{CH}$ in measure 4 via the passing tone $\mathrm{D}$, then the $\mathrm{E}$ of measure 1 over the first-inversion chord should be verticalized over the opening tonic in root position. And once we opt for such a consistent application of the rules, the choice of organizing the beginning of the melody 


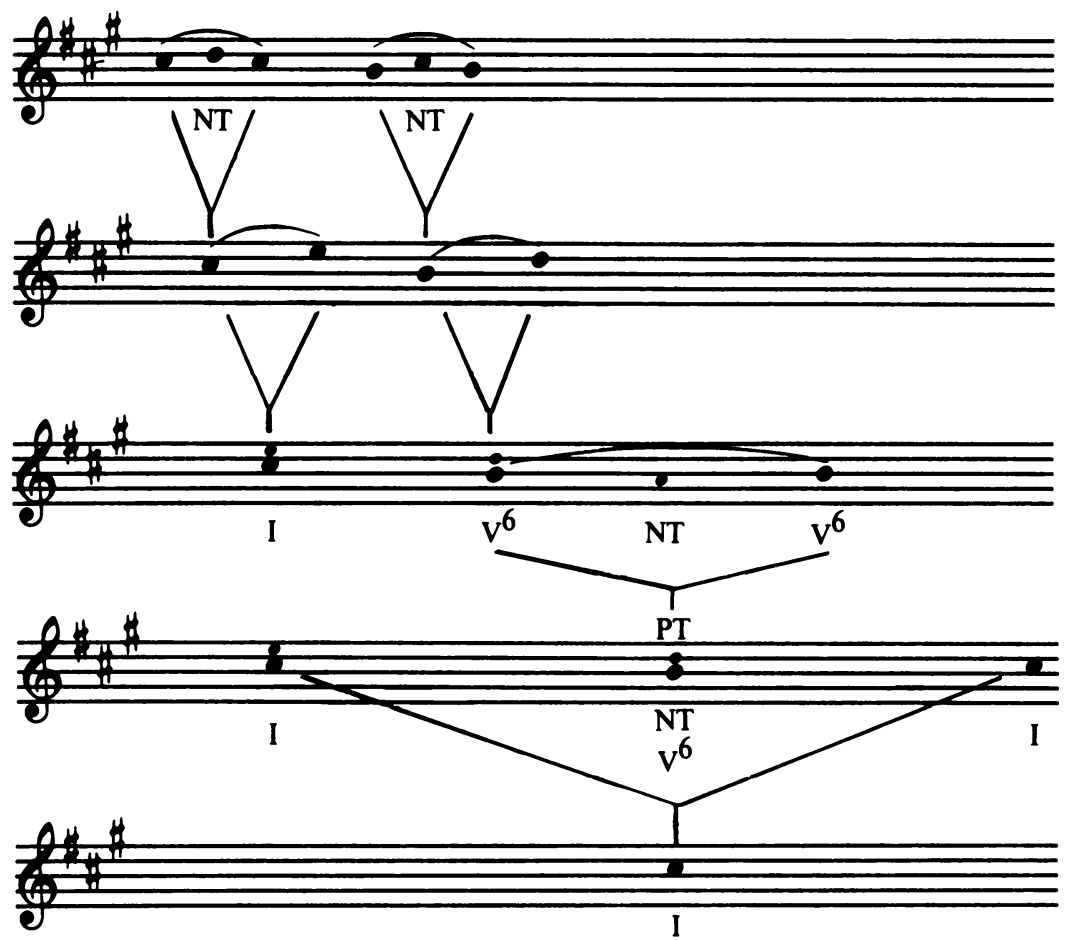

Figure 20.

on $3(\mathrm{CH})$ instead of 5 (E) appears much more convincing. Indeed, one suspects it was just such consistency of application in verticalizing the melodic notes of measures 1-2 that led Schenker to his decision to analyze the phrase as a descending 5-4-3-2 line instead of a 3-2 line.

Lester's analysis comes to grief on external grounds as well. For his decision to treat the melodic $A$ on the downbeat of measure 3 as a neighboring tone prevents his analysis from recognizing the $\mathrm{C}-\mathrm{B}-\mathrm{A}$ melodic line, so forcefully brought to the fore on the downbeats of measures 1,2 , and 3 . Likewise, because the same melodic $A$ is tagged on the lowest level as an ornamental tone in the service of the dominant $\mathrm{V}^{6}$ 's, and thus reduced out of the analytical picture early on, the analysis also cannot present the rising melodic line of the $A$ and $B$ in measure 3 to the $C \#$ of measure 4 . Of course, it could be asserted that in Schenkerian terms both these lines exist only on the very lowest level, that of the piece itself, and that that is where they should be dealt with analytically. But can it be doubted that these two lines occur structurally on higher levels?

Moreover, since Lester's avowed purpose is to show how tonal structures are articulated by form, his analysis of the first two bars seems all the more 
unacceptable inasmuch as Mozart's motivic repetition perceptually drums the $\mathrm{C}$-B-A and A-B-C melodic lines into our memory. Finally, it should be noted once again that in this tree-structured analysis in the Schenkerian vein, there is still no provision made for explaining the manifest contiguous melodic relationships of the $\mathrm{E}$ (measure 1 ) to the $\mathrm{B}$ (measure 2 ) or the $\mathrm{D}$ (measure 2) to the A (measure 3), other than to say they are voices of chords. ${ }^{75}$

Though Schenkerian theory is somewhat flexible in its application, despite a rigid adherence to the rationalistic premises of the Ursatz in generating levels from high to low, the problems raised by its tree-generated systemic levels thus cannot be circumvented by varying the Urlinie chosen. Both Schenker's 5-4-3-2 and Lester's 3-2 applied to the first four bars of Mozart's theme generate the same kinds of problems-completely decomposed levels, inconsistency as regards the interaction between parameters other than harmonic summarization, the omission of manifestly contiguous or empirically discontiguous relationships, the disregard of manifest conformance in the creation of levels, or the making of false formal relationships under the guise of illusory melodic lines derived rationalistically by an unswerving devotion to the a priori premises.

As opposed to network-generated hierarchies where formation, deformation, and transformation are at work vertically and horizontally, both up and down levels, in tree-generated levels the transformational rules are always of the either-or variety. This creates problems because music is not systemic but rather truly hierarchical. In a tree system, either we elect the A in measure 3 as an "inside voice" to the C\# of measure 4 and end up prolonging the tonic beginning with a structural 5 on the $\mathrm{E}$ in measure 1 , as Schenker did (Figure 18), or else we elect the $A$ in measure 3 as a neighboring tone and end up prolonging the transformed dominant $\mathrm{V}_{5}^{6}$ as a neighborpassing tone from a structural 3 beginning on the $\mathrm{C}$ in measure 1 , as Lester did (Figure 20). Neither solution is satisfactory. Schenker's choice of the parameter of voice-leading over that of harmony allows for both the $\mathrm{C}-\mathrm{B}-$ $\mathrm{A}$ and the A-B-C lines on the beats of measures $1,2,3$, and 4 , which is certainly desirable, but it comes at the expense of a rationalistically untenable 5-4-3-2 Urlinie. Lester's choice of elevating the return of the $\mathrm{V}^{6}$ chord over the parameter of voice-leading allows for a more convincing 3-2 Urlinie, but this comes at the expense of rationalistically conceiving the A of measure 3 as a neighboring tone between the B's of measures 2 and 3 .

In terms of the parameter-by-parameter analysis presented earlier (Figure

75. I shall pass over Lester's analysis of the D in measure 4. All of the criticisms I made about DeVoto's treatment of that tone as a mere neighboring note apply here. The concept of hierarchy, to repeat, makes such a reduction highly suspect. 
15 ), the view of each analyst is, however, partly right. The $\mathrm{C}-\mathrm{B}-\mathrm{A}$ line (on the beats of measures 1,2 , and 3 ) and the A-B-C\# line (measures 3-4) seen by Schenker transform because they initiate and terminate melodic processes created on higher levels according to the principles of near-registral return, the closural cumulation of duration, and the mimicry inherent in the form. Though the $\mathrm{V}^{6}$ 's seen by Lester (measures 2-3) emerge transformationally for the same reasons, so does the chord on the downbeat of measure 3 , if only transiently. In a melodic sense, the two $\mathrm{V}^{6}$ chords thus exhibit medial functions. Hence, as the recursive analytical progression in Figure 15 makes clear, there are arguments as to why the downbeat of measure 3 cannot be construed wholly in the way Lester thinks. Moreover, although Lester's 3-2 Urlinie seems correct overall, his nested neighboring tones on the $A$ (measure 3 ) and the $B$ (measure 2 ) seem both systemically rationalistic and analytically simplistic. Similarly, Schenker's 5-4-3 of the Urlinie makes little sense in light of the closural and nonclosural functions of the individual parameters (those of melody, harmony, duration, as defined earlier).

It might be argued that superimposing one of these analyses on top of the other-melding them together, as it were-would be one way of gaining a more complete understanding of how the musical materials interact in Mozart's phrase. And it is true that analyzing a piece from several vantage points is often enlightening. But such a compilation would never get us very far theoretically, for it would not force us to make transformation decisions on the basis of parametric interactions. Nor would it enable us to deal with vertical feedback and level-transience. Above all it would not force us to deal with, and thus revise, the fundamental difficulties of the theoretical system. At best, the eclectic rationale is a stopgap until serious attention can be directed toward fundamental problems. It seems far better to approach hierarchical analysis at the outset by conceptualizing musical events for what they really are in and of themselves: potentially both open and closed on the same level at the same time. Then we can go on to analyze level-events parameter-by-parameter with functions of low levels becoming embodied in the functions of higher levels. From this we can then attempt to hypothesize the rules by which formation, deformation, and transformation generate the vertical and horizontal networks inherent in the partially decomposed levels of musical hierarchies.

As asserted earlier, the epistemology of a given theory becomes known primarily through the examination of its analytical symbols rather than through the informal-often illusional-interpretations that graduallyand often unjustifiably-become associated with those symbols over the course of time. In the Schenkerian analyses we have considered, for instance, there is nothing in the symbology to represent the interaction of purely melodic, durational, or manifestly conformant properties as independent determinants of closure and nonclosure with respect to the emergence of 


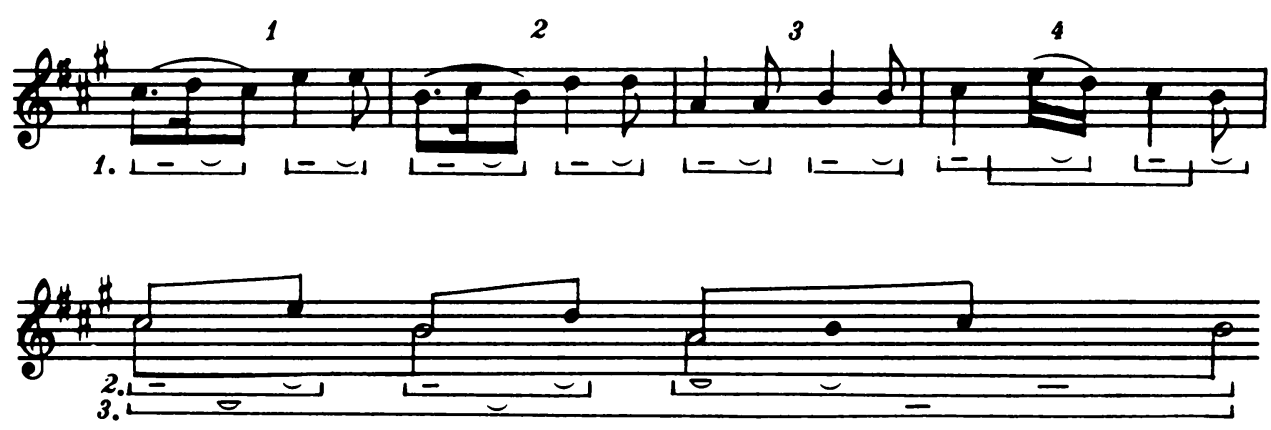

Figure 21. After Meyer.

levels. Nor is there anything in the symbology to suggest that the manifestly contiguous melodic relationships severed on account of the tree parsing even exist (e.g., the relationships from measures 1-2 or 2-3). But Schenkerian theory is not alone in this latter respect.

Let us examine very briefly one other analysis of the first phrase of Mozart's theme, paying close attention to the reductive-analytical symbology. The analysis in question is a good deal simpler than the three we have seen thus far, the analyst preferring to concentrate on the aspect of melody which, as we have seen in Mozart's theme, is the crux of the matter as far as horizontal and vertical networks are concerned.

The analysis is that of Leonard B. Meyer (Figure 21), who offers two reductions, one of which in important respects resembles DeVoto's and the other of which combines the best of Schenker and Lester. ${ }^{76}$ Unlike Schenker and Lester, however, Meyer essentially generates his melodic pitch structures not from anything like the Ursatz but rather from what he believes to be the summarizing rhythmic structure on each given level. ${ }^{77}$ Nevertheless, the result of Meyer's "rhythmic" hierarchy, like that of Schenker and Lester,

76. The first analysis is from Meyer (1973, p. 37). I have grafted onto the example the rhythmic analysis given on page 39 . Though I will criticize Meyer's analyses on the basis of what is implied in his analytical symbology-since I believe symbologies reveal the essence of any analytical approach-it should nevertheless be pointed out that Meyer never intended these two analyses of the Mozart phrase to be exhaustive, his purpose in the chapter of the book from which the Mozart examples come being to discuss the relationship of analysis to performance.

77. For Meyer's principles see his book (1973, pp. 121-123). 
is also a tree structure, as shown in the display:

(6)

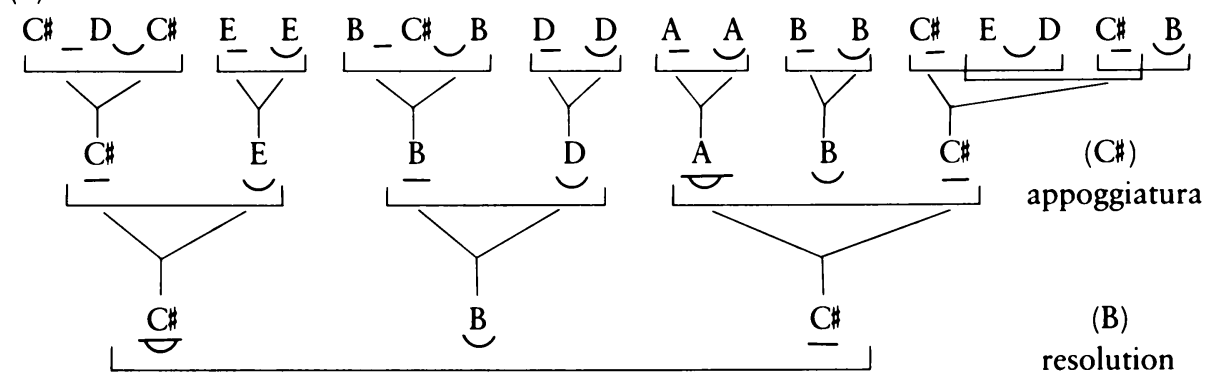

The structural pitches in Figure 21 are those falling on the accents $(-)$ on each level, the one exception being that of the B in measure 4 , which requires a special "appoggiatura rule," following the resolution of the B over the $\mathrm{V}^{78}$ Nonstructural tones are those occurring on nonaccents $(\smile)$. Each accent and nonaccent make up a form (shown by brackets), named by the order and number of the accents and nonaccents within the bracket in the traditional terms of prosody (trochee, iamb, dactyl, anapest, etc.). The "interface" (edge) of any given bracket represents closure, located more specifically by the relative positioning of accent and nonaccent within the bracket. The symbol $\checkmark$ shows that the pitch on that level was understood in prospect to be an accent but in retrospect a nonaccent. Groups may overlap or "pivot" ( $\longleftarrow \_$), as seen in measure 4 .

The basic technique for generating melodic structural tones would thus seem to differ from Schenker's top-down approach in that Meyer's analysis appears to work from the bottom up-from straightforward metric groups of trochees $(-\smile$ ) on the lowest level (level 1) to end-accented groups (anapests) on the higher levels (e.g., method is epistemologically similar to Schenker's in that there is a strong a priori bias toward certain foreordained patterns on higher levels-namely, end-accented groups (iambs, dactyls). ${ }^{79}$

Meyer is especially interested in understanding the relationship of process (or function), as shown in the note reductions on various levels, to rhythmic form, as symobolized by the brackets and the order of the poeticfeet symbols on various levels. Like Schenker's Ursatz, these rhythmic forms attempt to summarize the interaction between the musical variables (mel-

78. Meyer (1973, p. 122).

79. The "tendency toward end-accented groups" is stressed throughout Meyer's book (1973). 
ody, harmony, duration, dynamics, meter, orchestration, and so forth).$^{80}$ Unlike Schenker, Meyer's conceptual split between process and form tries to come to terms with the tree-network phenomenon of music. As we shall see, however, he is not wholly successful in this analysis since the generative technique of employing summarizing "rhythms" to determine pitch transformations in fact creates basically decomposed, and thus systemic, rather than hierarchical, levels. Let us look more closely.

In Figure 21, the white notes on the downbeats ( $\mathrm{C}$, B, and A)-exactly like those of DeVoto's meter-generated level $e$ (Figure 16) - are symbolized as being more important than the black notes $(\mathrm{E}$ and $\mathrm{D}$ on the nonaccents and $C \#$ ). The white-note $B$ at the end is structural because, as mentioned, it is the resolution of the 6 chord in measure 4 (and in this regard completely unlike DeVoto's level c). The B in measure 3 is thus the least consequential note of all, lying stemless between the $A$ and the $C \#$ because of its passingtone function. Thus, the three pairs of notes beamed together $(C H-E, B-D$, and $\mathrm{A}-\mathrm{C} \#$, ascending stems) reflect the rhythmic groupings and the brackets in which they appear on level 2.

But if theories are known by their analytical symbols, then there would seem to be one problem with this: namely, the inconsistency between the AC\# note reductions in measures 3-4 and the rhythmic analysis there. For in terms of the rhythmic symbols, the $\mathrm{CH}$ in measure 4-an accent on every level-is analyzed as a stronger tone than the $\mathrm{A}$ in measure 3 , an accent turned into a nonaccent $(\square)$. And if the correspondence between pitch reduction and rhythmic groupings is to mean anything, then in terms of closure the $\mathrm{A}$ in measure 3 should be the black note and the $\mathrm{C}$ the white note-either that or else the rhythmic analysis must be changed. As we have seen, problems of this sort typically arise in tree-structured generations because the complexities of musical relations are much too resistant to yield completely to such systemic summarizations.

Moreover, it is clear with reference to the symbology of the analysis that the similarity symbolized among the three pairs of notes (C\#-E, B-D, A$\mathrm{C}$ ) - whether beamed together as in Meyer's example or slurred together as in Schenker's broken thirds (Figure 17)-is misleading from a hierarchical point of view. For although the $C \#-E$ and $B-D$ pairs of measures 1 and 2 are highly conformant on low and middle levels, the A-C\# pair is significantly different from these since, to take just one aspect of the parameter of melody, the $C \sharp$ of measure 4 functions hierarchically as the termination of the ascending $\mathrm{A}-\mathrm{B}-\mathrm{C}$ process. In any hierarchical analysis that fact would have to be embodied within the $C \sharp$ of measure 4 which would prevent the note from creating an interval of a third comparable with the $\mathrm{C}-\mathrm{E}$ and $\mathrm{B}-$ $D$ pairs in measures 1-2. As we saw earlier in Schenker's analysis and see 


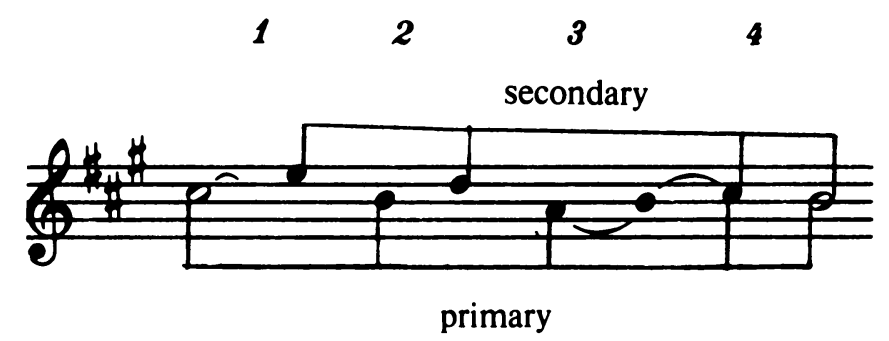

Figure 22. After Meyer.

here in this one, a tree-structured approach that generates completely decomposed levels tends to lead to false formal comparisons.

Meyer is clearly aware of this differentiation as his second analysis shows (Figure 22). ${ }^{81}$ Here he splits Mozart's theme into two strands, beaming together the notes on the downbeats as the primary line (the $\mathrm{C} \#-\mathrm{B}-\mathrm{A}-\mathrm{C} \#-$ $B$; note how the ascending $B$ between the $A$ and $C \#$ is omitted on the level of the bar because it is a nonaccent) and the notes on the nonaccents as the secondary line (the descending E-D-C $-B$ ). It would seem nothing would differentiate the $\mathrm{C}$ in measure 4 as a terminal tone more than the convergence of the two lines (double stemmed in the example)-thereby demonstrating how different the A-C\# pair is from the earlier $\mathrm{C}-\mathrm{E}, \mathrm{B}-\mathrm{D}$ pairs.

This analysis, however, is still not truly hierarchical because complete decomposability between certain melodic relations remains the rule. The stemless B in measure 3 of the analysis, for instance, is cut off from any structural participation at the level of the two strands even though, as we saw in Figure 15, it possesses a significant measure of both durational and harmonic closure, fully as much as-nay, more than-the D of measure 2. Furthermore, if one reduces the $B$ in measure 3 out of the analysis because it is a passing tone, then in order to be consistent one also must do the same for the $\mathrm{D}$ in measure 2 (passing between the $\mathrm{E}$ and the $\mathrm{C}$ in the secondary line) since, as a nonaccent on the level of the bar, it functions de facto at the same level.

If on the other hand one argues that the $\mathrm{D}$ in measure 2 should be left in the analysis on this level (beamed between the E-C\# descent from measure 1 to measure 4 ) because of its conformant functional similarity to the $\mathrm{E}$ as a nonaccent ending a trochee, then one must also admit to the formal similarity between the $\mathrm{A}$ and $\mathrm{B}$ of measure 3 where on the beat-level both tones are 
accents of trochees (recall also Figure 14). The point is, in order to be consistent the tree-parsed relations generated via the rhythmic groups of poetic feet should have made the B in measure 3 structural on some level, presumably the same level as that of the $\mathrm{D}$ in measure 2.

I raise these issues of analytical symbology, of course, not to nitpick but to emphasize again that adherence to a systemic-analytic tree to generate levels will tend to lead to theoretical and empirical inconsistencies since music is inherently hierarchical. A close examination of symbology, moreover, forces us to sharpen our parametric rules for transformation. What would lead one, for instance, to pick out the D of measure 2 as structural, but omit the B of measure 3? It can't be harmony since the $D$ is more dissonant (over the $\mathrm{V}_{3}^{4}$ ) than the $\mathrm{B}$ (over the $\mathrm{V}^{6}$ ). It can't be voice-leading since both the $\mathrm{B}$ and $\mathrm{D}$ function as passing tones on the same formal and metric level (both on beat 2) if Meyer's analysis is accepted. It can't be conformance since the $\mathrm{D}$ in measure 2 is as much like the $\mathrm{E}$ of measure 1 preceding it as the $\mathrm{B}$ in measure 3 is like the A preceding it. What then? Would one be satisfied to weight the near registral return of the $D$ over the harmonic resolution of the $B$ ? Would one be justified in elevating this discontiguous registral relationship affecting the $\mathrm{D}$ over the contiguous harmonic relationship affecting the $B$ ?

Meyer's systemic tree, like Schenker's and Lester's, also completely decomposes certain other important melodic relations in Mozart's theme. Again one wonders, for instance, about the omission of the contiguous relations between the E-B (measures 1-2) and the D-A (measures 2-3). Meyer, however, recognizes the consequences following from his analysis: he says outright that the "interval from E to B across the bar must be an unrealized perfect fourth" and thus that it is "not perceived as an active syntactic connection." 82

Rhythmically, the connective relationship between this $\mathrm{E}$ and $\mathrm{B}$ is also omitted since the symbology of the bracket ending on the eighth-note $E$ in measure 1 and the new bracket beginning on the quarter-note $B$ in measure 2 shows a "space" (an "interface") between those two tones. The same is true of the D in measure 2 and the A in measure 3 . Such complete decomposability stems, as we have seen, from the tree-structured systemic generation of levels, but is it tenable?

It seems highly doubtful to me that the ear does not hear those intervals

82. Meyer (1973, p. 37). Similar remarks asserting such disconnection have been made by Salzer (1962). In light of these examples there can be no doubt that our theoretical beliefs and analytical conclusions substantively influence what we think we hear. Note, incidentally, that on the basis of the symbology of my own analysis of the Mozart, one would also have to conclude that contiguous relations are omitted since my analysis does not deal with the manifest intervallic relations. 
as fourths, that is to say, hear them as in fact being "realized." Furthermore, it seems implausible to assert that there is no "active syntactic connection" in these instances since the countercumulative rhythm on the $E$ at the end of measure 1 leads that note quite naturally to the cumulative $B$ in the next bar. ${ }^{83}$

Because in true hierarchies both a priori and a posteriori summarizations are always subject to vertical feedback and thus deformational revision, we would not, of course, have to adopt such an either-or analytical position since in conceptualizing partially decomposed levels both the connection and the articulation between these notes would have to be admitted. The closure in one parameter-say, the effects of conformance-would not preclude the nonclosure of another-say, the countercumulative duration. The connection between the eighth-note $E$ at the end of measure 1 and the quarter-note $B$ at the beginning of measure 2 might be weakened because of the occurrence of the near-return stretching back to the $\mathrm{C} \#$ opening measure 1 , but it would still be "there" nonetheless as part of a vertically networked hierarchical complex.

\section{Conclusion: Ranking vs. Level; Synthesis vs. Analysis}

What a strange field music theory is at this juncture in its history. One would have thought there could be no less controversial example for analysis than the first four bars of Mozart's little theme. Yet Meyer finds one 54-3-2 line, Schenker another. Lester's avowed Schenkerian approach agrees not with Schenker but with Meyer about the existence of a 3-2 structure. Lester argues for a series of nested neighboring tones instead of the descending and ascending lines of Meyer and Schenker. DeVoto agrees with Meyer about the metric $\mathrm{C}-\mathrm{B}-\mathrm{A}$ in measures 1-3 but, unlike Meyer as well as unlike Schenker and Lester, dismisses the B over the articulating half cadence $\mathrm{V}$ in measure 4 from the schema very early-as a mere neighboring tone.

All these analytical differences-and they are not as trivial as one might have supposed at the outset-result, I submit, from the fact that levels exist in artifactual phenomena only as partially decomposable events. To conceive level-relationships in music as essentially a tree system of completely decomposable parts can only lead to unsatisfactory and erroneous analyses. Musical hierarchies are inherently networked both vertically and horizontally. It is just such complexity that makes the formation of music theory so difficult.

For these reasons, I see no alternative-if music theory is to advance- 
than to develop analytical theories that avoid systemic summarizations, eschewing level-generation from a priori transforms. We must work to generate analyses where the low-level events that transform to higher levels carry with them all their original melodic, harmonic, and durational properties and all their low-level parametric functions. And though it cannot be denied that traditional systemic approaches are worthwhile in bringing into sharp symbologic focus important analytical anomalies and important theoretical disagreements-e.g., those concerning the construction of measure 3 in the Mozart example-I see no viable alternative for the future of hierarchical theory in music other than to develop an analytical methodology based on evaluating all the parameters of music separately. ${ }^{84}$

One final point: we tend to think of the concept of hierarchy mostly in terms of levels. But levels in fact are primarily rationalistic concepts, regardless of how refined. There is, however, another important meaning of the term hierarchy that we should not overlook-that of ranking. The aspect of ranking emphasizes that in true hierarchies individual events on low levels are never assimilated completely into high levels because low-level functions literally produce higher-level transformations, whether permanent or transient. High levels in real hierarchies incorporate lower-level functions but do not absorb them. Even when exogenous (extraopus) or endogenous (intraopus) style is called upon in analysis, its invocation depends literally on what happens generationally on the note-to-note level. To be sure, there is constant feedback between the style the listener has learned and the idiostructure presented in the piece. But it is the work that triggers our perceptual invocation of the relevant style, not vice versa. Artworks are artworks because they constantly resist assimilation-and thus analytical reduction-into the style.

In light of the issues stressed in this paper, the idea of individual events being hierarchically ranked thus reminds us that the theorist's work is not done until analysis leads back to synthesis. Whether the parsing of musical parameters into levels or stages of levels is offered as a heuristic strategy or whether deconstruction is thought to lead to a symbological representation of the real perceptual-cognitive world, any analysis of partially decomposed levels implies a recomposed, reconstructed synthesis.

Moreover, whatever the discipline, all real theories ultimately aim for, or should aim for, an analytical precision that allows for, or at least implies, measurement. In the case of musical hierarchies, that implied measurement attempts to define the relative degrees of structural rank.

Figure 23, which is based on the analysis of transformational levels

84. I have attempted to show one way this might be done in my article (Narmour, in press). 


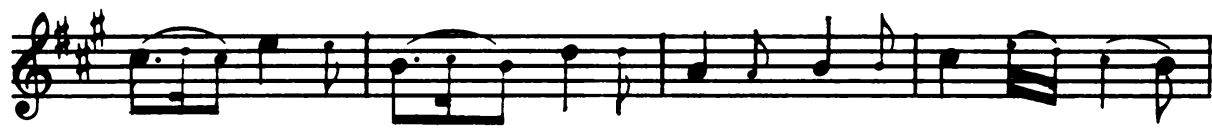

Figure 23.

presented earlier in Figures 12 and 15, displays in rough form a structural ranking of the nineteen melodic events of the Mozart melody by presenting their level-structures in terms of different sizes of note heads, synthesized onto one staff. (From the two detailed analyses, it was logically easy to rank each note in terms of its ratio of closure to nonclosure and then group it according to one of the four sizes of note heads.) ${ }^{85}$ The example does not represent an idiostructural symbology, of course, since in an idiostructural representation each note would be a different size, so to speak. But it shows how an idiostructural synthesis would proceed if nonrecurrent rules of closural and nonclosural differentiation were known.

Figure 23 implies (and only implies) what lies behind, or should lie behind, the hierarchical concept. Refining that approach must be a major goal of music theory if we are ever to understand more fully how artworks are perceived in all their stylistic and idiosyncratic glory. ${ }^{86}$

\section{References}

Ayer, A. J. Language, truth and logic. New York: Dover Publications, 1946.

Cone, E. T. Musical form and musical performance. New York: W. W. Norton, 1968.

Cooper, G., \& Meyer, L. B. The rhythmic structure of music. Chicago: University of Chicago Press, 1960.

Ellis, J. M. The theory of literary criticism: A logical analysis. Berkeley: University of California Press, 1974.

Hasty, C. F. Review of Piston's Harmony. Journal of Music Theory, 1982, 26, 155-165.

Lerdahl, F., \& Jackendoff, R. Toward a formal theory of tonal music. Journal of Music Theory, 1977, 21, 111-171.

Lester, J. Articulation of tonal structures as a criterion for analytic choices. Music Theory Spectrum, 1979, I, 67-79.

Meyer, L. B. Explaining music. Berkeley: University of California Press, 1973.

Morgan, R. P. The theory and analysis of tonal rhythm. Musical Quarterly, 1978, LXIV, 440-451.

Narmour, E. Beyond Schenkerism: The need for alternatives in music analysis. Chicago: University of Chicago Press, 1977.

85. Throughout the relatively short history of musical notation, it may be recalled that there have been occasional nonsystematic attempts to represent structural weight in the sizes of the manifest notes themselves (e.g., grace notes, appoggiaturas, diminution, etc.).

86. I wish to thank my colleagues George Crumb and Leonard B. Meyer for their help in writing this article. 
Narmour, E. Toward an analytical symbology: The melodic, harmonic, and durational functions of implication and realization. Proceedings of the International Conference on Musical Grammars and Computer Analysis, in press.

Pattee, H. H. Postscript. In H. H. Pattee (Ed.), Hierarchy theory. New York: George Braziller, Inc., 1973.

Piston, W. Harmony, revised and expanded by Mark DeVoto. New York: W. W. Norton, 1978.

Quine, W. V. From a logical point of view. New York: Harper \& Row, 1963.

Salzer, F. Structural hearing. New York: Dover Publications, 1962.

Schenker, H. Der freie Satz. Vienna: Universal Edition, 1956.

Simon, H. A. The sciences of the artificial. Cambridge, Mass.: M.I.T. Press, 1969. 